\title{
Cryo-vacuum testing of the JWST Integrated Science Instrument Module
}

\author{
Randy A. Kimble*a, M. Begoña Vila ${ }^{\text {b,a }}$, Julie M. Van Campen ${ }^{\mathrm{a}}$, Stephan M. Birkmann ${ }^{\mathrm{c}}$, Brian J. \\ Comber $^{\text {d,a }}$, Curtis C. Fatig, ${ }^{\mathrm{e}, \mathrm{a}}$, Alistair C. H. Glasse ${ }^{\mathrm{f}}$, Stuart D. Glazer ${ }^{\mathrm{a}}$, Douglas M. Kelly ${ }^{\mathrm{g}}$, \\ Steven D. Mann ${ }^{\text {h,a }}$, André R. Martel ${ }^{\mathrm{i}, \mathrm{j}}$, Kevin J. Novo-Gradac ${ }^{\mathrm{b}, \mathrm{a}}$, Ray G. Ohl ${ }^{\mathrm{a}}$, Konstantin I. \\ Penanen $^{\mathrm{k}}$, Scott O. Rohrbach ${ }^{\mathrm{a}}$, Joseph F. Sullivan ${ }^{1}$, Dean Zak ${ }^{\mathrm{m}}$, Julia Zhou ${ }^{\mathrm{n}}$ \\ aNASA's Goddard Space Flight Center, 8800 Greenbelt Rd, Greenbelt, MD 20771 \\ bStinger Ghaffarian Technologies, 7701 Greenbelt Rd \#400, Greenbelt, MD 20770 \\ ${ }^{\mathrm{c}}$ European Space Agency - STScI, 3700 San Martin Dr, Baltimore, MD 21218 \\ ${ }^{\mathrm{d} C}$ Comber Thermal Solutions, 8367 Silver Trumpet Dr, Columbia, MD 21045 \\ ${ }^{\mathrm{e}} \mathrm{AS}$ and D LLC, 7000 Muirkirk Meadows Dr, Beltsville, MD 20705 \\ ${ }^{f}$ UK Astronomy Technology Centre, Edinburgh EH9 3HJ, United Kingdom \\ ${ }^{\mathrm{g}}$ Steward Observatory, University of Arizona, Tucson, AZ 85721 \\ ${ }^{\mathrm{h}}$ the Hammers Company, 7500 Greenway Center Dr \#1500, Greenbelt, MD 20770 \\ iSpace Telescope Science Institute, 3700 San Martin Dr, Baltimore, MD 21218 \\ ${ }^{j}$ NRC Herzberg Astronomy and Astrophysics, 5071 West Saanich Rd, Victoria, BC, Canada \\ kJet Propulsion Laboratory, 4800 Oak Grove Dr, Pasadena, CA 91109

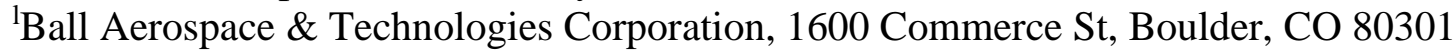 \\ ${ }^{\mathrm{m}}$ CSRA, 3700 San Martin Dr, Baltimore, MD 21218 \\ ${ }^{\mathrm{n}}$ Honeywell Aerospace, 303 Terry Fox Drive, Suite 100, Ottawa, ON K2K 3J1, Canada
}

\begin{abstract}
In late 2015/early 2016, a major cryo-vacuum test was carried out for the Integrated Science Instrument Module (ISIM) of the James Webb Space Telescope (JWST). This test comprised the final cryo-certification and calibration test of the ISIM, after its ambient environmental test program (vibration, acoustics, EMI/EMC), and before its delivery for integration with the rest of the JWST observatory. Over the 108-day period of the round-the-clock test program, the full complement of ISIM flight instruments, structure, harness radiator, and electronics were put through a comprehensive program of thermal, optical, electrical, and operational tests. The test verified the health and excellent performance of the instruments and ISIM systems, proving the ISIM element's readiness for integration with the telescope. We report here on the context, goals, setup, execution, and key results for this critical JWST milestone.
\end{abstract}

Keywords: JWST, ISIM, infrared, integration and test, cryo-vacuum testing

\section{INTRODUCTION}

\subsection{Introduction to the ISIM}

The Integrated Science Instrument Module (ISIM) for the James Webb Space Telescope (JWST) is comprised of four scientific instruments (SIs) and various structural, thermal, electronic, and operational systems that support them. The SIs are: the Near-Infrared Camera (NIRCam) ${ }^{1,2}$, the Near-Infrared Spectrograph (NIRSpec) $)^{3,4}$, the Mid-Infrared Instrument (MIRI) $)^{5,6}$, and the Fine Guidance Sensor/Near-Infrared Imager and Slitless Spectrograph (FGS/NIRISS) ${ }^{7,8}$. Within the latter module are both a science channel (NIRISS) and a pair of Fine Guidance Sensor channels which, as the name implies, provide pointing error signals on selected guide stars to the attitude control system for the observatory. See also Greenhouse $(2016)^{9}$ for an overview of the capabilities and status of this powerful suite of instruments.

*randy.a.kimble@nasa.gov; phone 1301 286-5783

Space Telescopes and Instrumentation 2016: Optical, Infrared, and Millimeter Wave, edited by

Howard A. MacEwen, Giovanni G. Fazio, Makenzie Lystrup, Proc. of SPIE Vol. 9904,

990408 - (c) 2016 SPIE · CCC code: 0277-786X/16/\$18 - doi: 10.1117/12.2231554

Proc. of SPIE Vol. $9904990408-1$ 
Additional ISIM systems include: 1) an optical metering structure to hold the instruments, made of composite material with highly stable performance through repeated cryo-cycles, 2) the electrical harnesses, 3) the Harness Radiator (HR) system, which cools the harnesses as they travel to the ISIM cold section from a set of warm electronics boxes, 4) the ISIM Electronics Compartment (IEC), which holds those warm boxes, 5) the cryogenic thermal control system, including high-conductivity heat straps and the locally-mounted elements of the mechanical cryocooler for the MIRI, 6) the ISIM Command and Data Handling system (ICDH), which is mounted in the separate spacecraft element of the observatory, 7) the Flight Software System, and 8) the Operations Scripts System that will control the event-driven operations of the instruments in flight. The instruments and some of these supporting systems are shown in Figure 1.

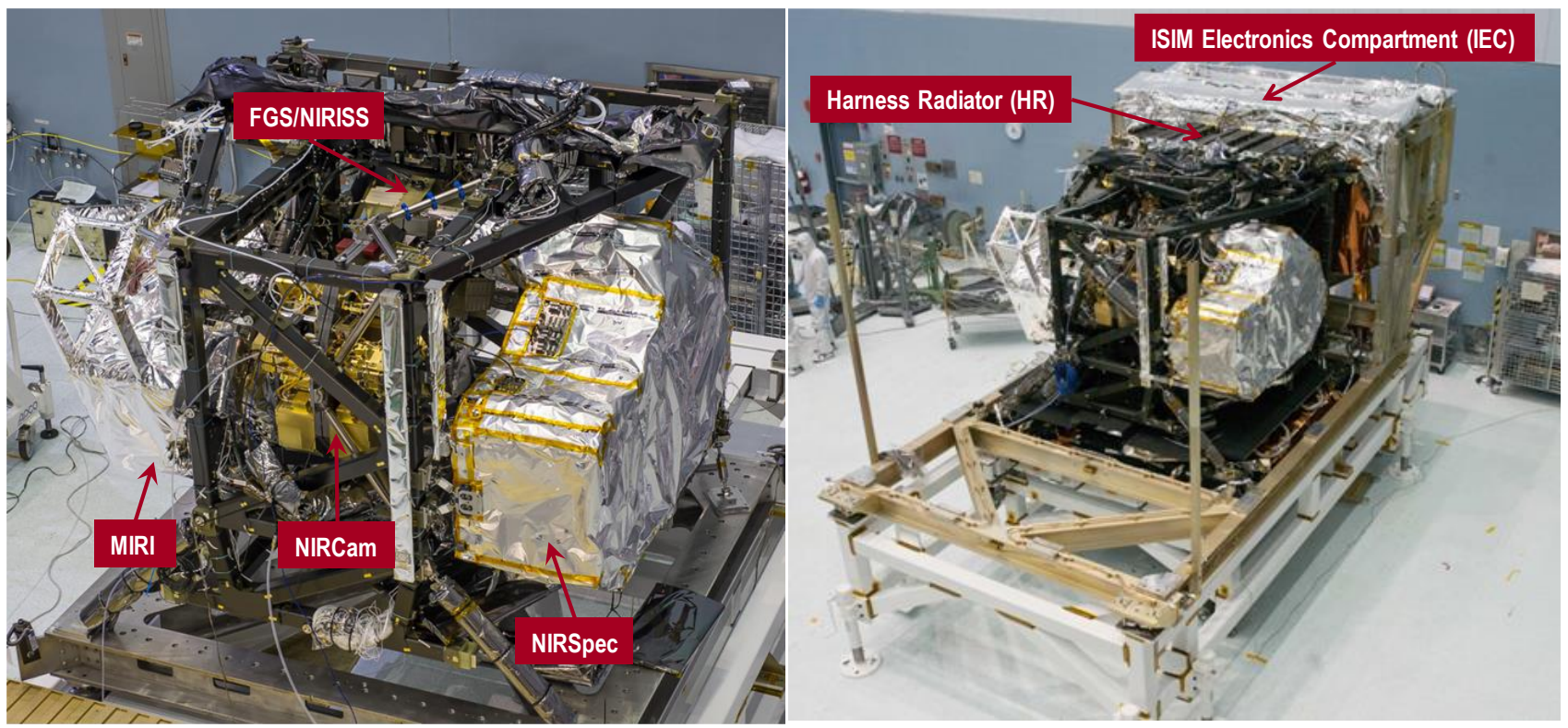

Figure 1. Left) The four ISIM SIs, mounted to a highly stable composite metering structure. This region operates at $\sim 40 \mathrm{~K}$. Right) The ISIM assembly during the buildup for the cryo-vacuum test. In addition to the SIs, the Harness Radiator (HR) can be seen. The ISIM Electronics Compartment (IEC) is hidden from view inside GSE cryopanels.

Integration of these systems into their intended final flight configuration was completed at NASA's Goddard Space Flight Center (GSFC) during 2015. The ISIM then underwent an environmental test program, culminating in a final predelivery cryo-vacuum verification and calibration test. That final, highly successful test is the focus of this paper.

\subsection{The path to the final ISIM-level cryo-vacuum test}

The path to the final ISIM-level cryo-vacuum test is depicted in Figure 2. This diagram shows schematically the stages of the ISIM integration and test program from the first cryo-vacuum test through the third and final cryo-vacuum test.

By the fall of 2013, the first two instruments to be delivered (MIRI and FGS/NIRISS) had been integrated to the ISIM structure and electrical systems. At that time, a preliminary cryo-vacuum test, called "CV1-RR" (Cryo-Vac \#1, RR for "risk reduction") was conducted to check out the facilities and procedures that had been developed for the ISIM test program. This test was very successful, despite the interruption of a U.S. federal government shutdown that enforced a lengthy "safe hold" during cryo operations and caused a descoping of some test activities. CV1-RR validated the basic soundness of the test approach, while providing valuable lessons for improving operations.

The remaining instruments, heat straps, and electrical systems were then integrated by the summer of 2014, after which a lengthy (116 days!), comprehensive, second cryo-vacuum test campaign ("CV2") was executed, demonstrating the health and performance of the instruments and other subsystems, and providing a thorough performance baseline before the ISIM underwent the rigors of the remaining environmental test program. In CV2, all critical test goals were accomplished.

Before that environmental test program was run, however, the opportunity was taken to make significant "final" upgrades to the instruments. While it is never certain that something is "final" until it is launched, it was the definite 
intention of this activity to put the instruments and all other ISIM hardware into the configuration that they will maintain through the remainder of ground testing and into flight operations.

The most notable upgrades were in the area of detectors for the near-infrared SIs. A vulnerability to dark current degradation in the H2RG HgCdTe Sensor Chip Assemblies (SCAs) had been recognized several years earlier and resolved through the fabrication of new arrays with a more robust design ${ }^{10}$. Accordingly, a full complement of new SCAs were installed into the FGS, NIRISS, and NIRSpec channels at this time. For NIRCam, the improved replacement arrays had been available early enough to go through the earlier CV2 campaign; an issue with one SCA in a ShortWave Channel four-SCA mosaic focal plane assembly was discovered during that test. In replacing that device, two others in the mosaic were damaged, so NIRCam ultimately required replacement of three of its total of ten SCAs during this upgrade activity.

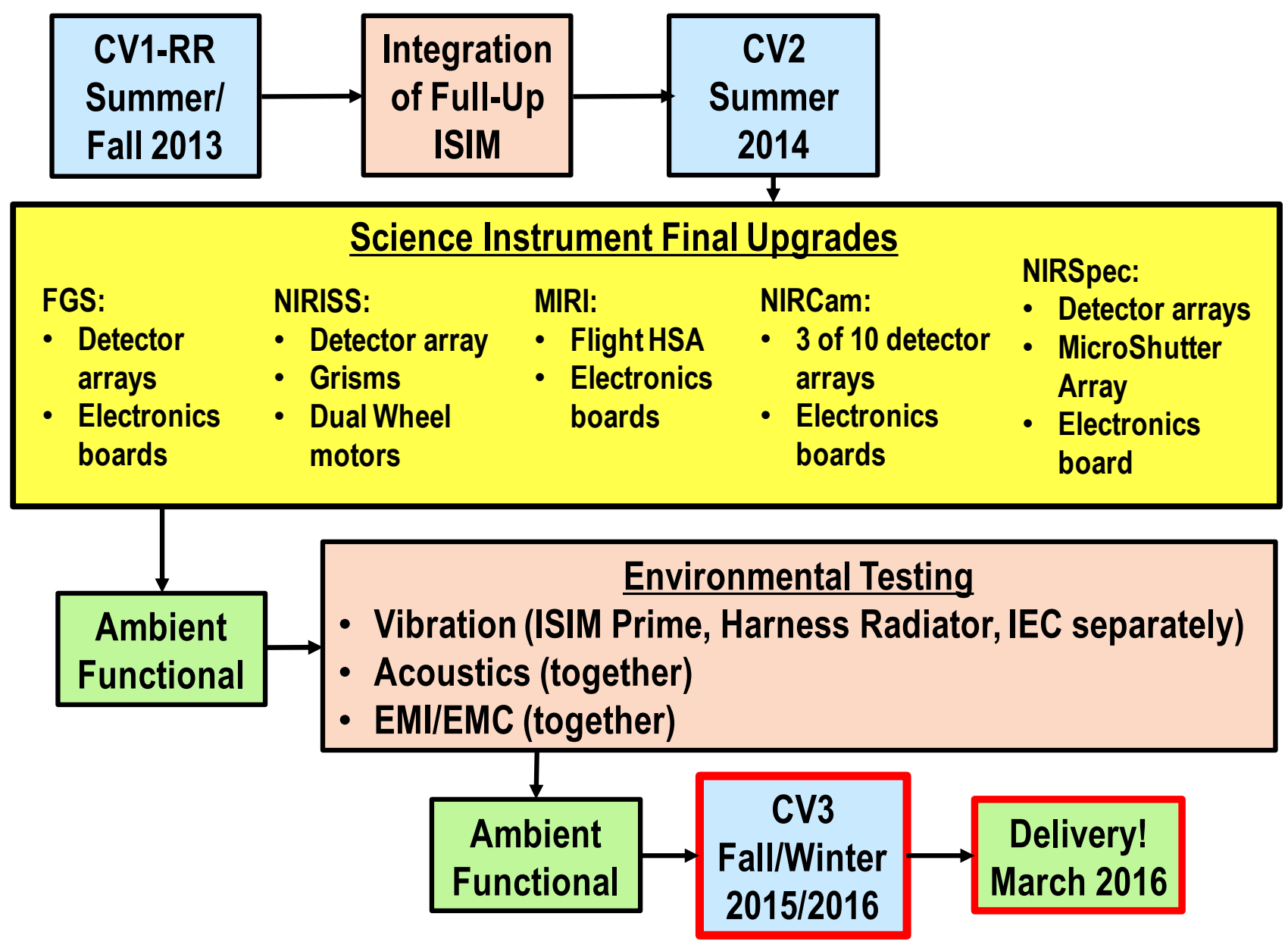

Figure 2. Schematic diagram of the latter stages of the integration and test flow for the ISIM element of JWST, leading to the final cryo-vacuum test, CV3, discussed in this paper, and delivery of the ISIM for integration with the telescope and ultimately the rest of the observatory. Three cryo-vacuum tests (CV1-RR, CV2, and CV3) with flight instruments were executed in total, as described in the text. ISIM Prime refers to the cold region of the ISIM element - i.e. the mounting structure with the instruments and local thermal hardware and harnesses, as distinct from the transitional Harness Radiator and the warm ISIM Electronics Compartment.

Other noteworthy upgrades within the instruments' optical assemblies included: 1) new MicroShutter Arrays for the NIRSpec, with a lower percentage of "failed-closed" shutters and greater robustness against acoustic damage $\left.{ }^{11}, 2\right)$ two 
new grisms within the NIRISS, one replacing a delaminated unit, and one providing a roughly $2 \mathrm{x}$ increase in throughput, and 3) improved drive motors for the filter and pupil (Dual Wheel) mechanism within the NIRISS. In addition, in the cold ISIM Prime region, the flight Heat Exchanger Stage Assembly (HSA) for the MIRI cryocooler replaced the engineering model unit used during CV1-RR and CV2, and modifications were made to the thermal joints where the heat straps mate to the ISIM and SIs, in order to improve the stability of those interfaces. Finally, within the warm IEC, improvements to various electronics boards for either performance or reliability were made for all SIs.

Once all of these upgrades were made, the instruments were re-integrated to the ISIM structure, to the HR and reintegrated IEC, and an ambient functional test was carried out, confirming all aspects of system health and performance that can be checked at room temperature - several ISIM systems, intended for cryogenic operation, cannot be operated at room temperature, and of course the detectors, while operable under ambient conditions, do not provide unsaturated image data until cooled.

The ISIM was therefore in its intended flight state as it went through the various ambient environmental tests (vibration, acoustics, EMI/EMC [electromagnetic interference/electromagnetic compatibility]) and then into its final cryo-vacuum verification test, CV3. An additional important aspect of the test program was to confirm the optical stability of the SIs and ISIM through the simulated launch environment. This needed to be done through comparison of image data taken during CV3 vs. during the pre-environmental CV2 campaign. Accordingly, it was critical that replacements of detectors and other optical components, and the remounting of the SIs and ISIM structure, be done with extremely precisely known positioning, so that replacement shifts would not be erroneously interpreted as shifts under vibration. Great attention was paid to optical metrology during the upgrade campaign, yielding replacement accuracies that could be assimilated within the required stability budgets.

\subsection{Objectives of the CV3 cryo-vacuum test campaign}

With CV3 as the final activity before delivery of the ISIM to integration with the telescope, CV3 thus constituted a huge milestone in the ISIM program. It was intended to provide the cryo-verification for the majority of ISIM-level requirements. It also provided the final verification/calibration opportunity with the OSIM telescope simulator (described in the following section) and its slightly better than flight-like image quality and flat-field sources. Finally, CV3 was the final test for which there would be any reasonable access to the instruments afterwards. While it would have been a serious matter to have to get into the instruments in the cold ISIM Prime region after CV3 (and no such need has arisen!), it would be much more problematic to have to do so after the telescope-level cryo-testing that is scheduled for next year - once ISIM is mounted to the telescope, access to the instruments becomes much more challenging and time-consuming. Hence, it was essential to have a thorough and successful cryo-test campaign before ISIM delivery.

The top-level objectives of the CV3 test were expressed in one sentence at the CV3 Test Readiness Review: "Verify the ISIM System in its final configuration, after environmental exposure, and provide a post-environmental performance baseline - including obtaining critical ground calibrations needed for science data processing in flight." We elaborate below.

The highest priority for CV3 was given to the following test activities: 1) The activity provides basic health and safety information, reducing the risk of finding a problem that could have a large impact if not found until the next test phase. 2) The activity verifies requirements that are susceptible to changes as a result of environmental testing (such as the optical stability through launch-like vibration noted above), and thus require comparison between pre- and post-environmental test data. 3) The activity provides data needed to close previous Problem Reports and Problem Failure Reports (i.e. collects the evidence that previously identified hardware and software issues had been successfully resolved). Completion of these categories of tests was considered absolutely essential for CV3.

However, for this final ISIM-level test, there were many other important test objectives, including: 1) Verify ISIMlevel or subsystem-level requirements. Requirements verification is of course a fundamental part of the test campaign - and the significant rework in some areas of the instruments dictated that some instrumental-level requirements be reverified, such as detector noise performance or optical alignment.

2) Obtain critical Model Validation data. ISIM thermal model correlation is a key example. Many ISIM thermal requirements are verified not directly by test, but by analysis, in observatory-level thermal models built up from lowerlevel models that have been validated by test, through the variety of thermal balance cases and heat flow measurements made in the test programs. Similarly, having a validated optical model for the NIRSpec is critical, given the complexity of the mapping of the programmable microshutter slits to the focal plane in the various spectral modes - after 
replacement of the microshutters and detectors in that instrument, acquisition of the calibration data to update the NIRSpec optical model was a critical CV3 goal.

3) Obtain data for calibration of on-orbit science observations. As noted, there were new detector arrays in all three near-infrared SIs - these required new dark exposures, flat fields, and pixel-by-pixel linearity measurements. The latter two measurements were obtainable in CV3 with the OSIM telescope simulator, but will not be obtainable in telescopelevel testing, which does not offer the flat-field illumination capability of the OSIM. Sky flats can ultimately be derived for broad filters in flight, but this takes a long time after launch and can never be done adequately in the narrower band filters. The MIRI instrument did not change detectors, but did have significant upgrades to the readout electronics chain, requiring that similar calibration data be obtained. The new NIRISS grisms required wavelength calibrations.

4) Gain experience for in-flight operations. There are several aspects to this. One is providing formal certification of software (so-called "level 3" certification of running on the flight hardware). Another is simply gaining run-time and usage experience for the operational/software products (such as using the Operations Script Subsystem [OSS] or flight CECIL command scripts as much as was practical). The critical operation of Guiding was extensively exercised, and the Fault Management system was also tested. Finally, some specific demonstrations were made of observing modes that will be important in flight operations: e.g. wavefront sensing and control demonstrations using the relevant hardware in the NIRCam, high $\mathrm{S} / \mathrm{N}$ radiometric stability tests (relevant to exoplanet transit spectroscopy observations), or observing with the NIRISS Non-Redundant Mask.

Prior to the test, a bottoms-up timeline of activities was developed, controlled by a Master Procedure and numerous SubProcedures, to accomplish these goals. Recognizing that it might not be possible to execute all planned activities, careful thought was given to the prioritization of test activities. Fortunately, as will be described more thoroughly below, no significant descopes of planned activities had to be made in executing CV3, which accomplished essentially all of the objectives outlined above.

\section{CV3 TEST CONFIGURATION}

\subsection{Schematic of the test configuration}

The CV3 test program was executed using the largest vacuum chamber available at NASA's Goddard Space Flight Center. The layout of the test hardware, highlighting some of the key elements of the system, is shown in Figure 3. The chamber is equipped with a nitrogen-cooled shroud enclosing most of its volume. Within that is mounted a gaseous Hecooled shroud developed for the JWST program, which allows the upper half of the chamber to be cooled to temperatures required for flight-like ISIM operations.

Within that upper region, the flight ISIM Prime (cold region), the Harness Radiator assembly, and the ISIM Electronics Compartment were mounted. ISIM Prime was surrounded by helium-cooled cryo-panels that emulated the environment expected during flight operations in the observatory. The Harness Radiator dissipated heat to a nearby cold sink, and the IEC was located within a separate set of helium cryo-panels, within which was also a high-capacity nitrogen-cooled sink located above the IEC radiator panel to absorb most of the $\sim 200 \mathrm{~W}$ dissipated by the IEC, to keep that high heat load away from the He cooling system, which has much less cooling capacity than the nitrogen system. In flight, the IEC power is radiated away from the observatory to deep space, with a highly directional radiator that was successfully tested in separate, earlier cryo tests. See Glazer and Comber $(2016)^{12}$ for further details of the thermal configuration.

An opening in the floor cryopanel below the ISIM admits light from a versatile, highly capable telescope simulator mounted below, within nitrogen-cooled panels. This simulator, called the OSIM (for OTE [Optical Telescope Element] Simulator) is shown schematically in Figure 3. A clever optical design (utilizing a single spherical mirror, a variety of fiber-fed sources located specifically for each instrument channel, a pupil-stop translation mechanism, and a tiltable fold mirror) allows the OSIM to deliver to each instrument channel a beam at the appropriate flight-like position, chief ray angle, f/\#, and pupil position, with an acceptably low and well-calibrated wavefront error, from a package that is elegantly compact (at least when compared with the $6.5 \mathrm{~m}$ diameter of the actual JWST telescope!) ${ }^{13}$. The OSIM operates at a temperature of $100 \mathrm{~K}$, though there are some localized electronics systems that run warmer.

It is of course vital that the OSIM faithfully mimic the principal optical properties of the real JWST OTE. Meticulous measurements to calibrate the OSIM and demonstrate the fidelity of its optical simulation were carried out in two separate precursor OSIM cryo-vacuum test programs before the flight ISIM tests ${ }^{14}$. The OSIM also contains extensive instrumentation for self-calibration, including an Alignment Diagnostic Module viewing through a central hole in the 
primary mirror, and a Monitor and Calibration Assembly that mounts within the ISIM during ISIM testing. These modules are used to verify the alignment and wavefront performance of the OSIM during each cryo test ${ }^{14}$. Despite the fact that the OSIM structure is made of aluminum, such that the delivered beam shifts by tens of mm during cooldown, the OSIM proved to be spectacularly repeatable and reliable throughout the ISIM test campaigns. Critical to that performance was the fact that the OSIM was never moved after its final cryo-calibration campaign. It remained fixed in place throughout the ISIM CV program.

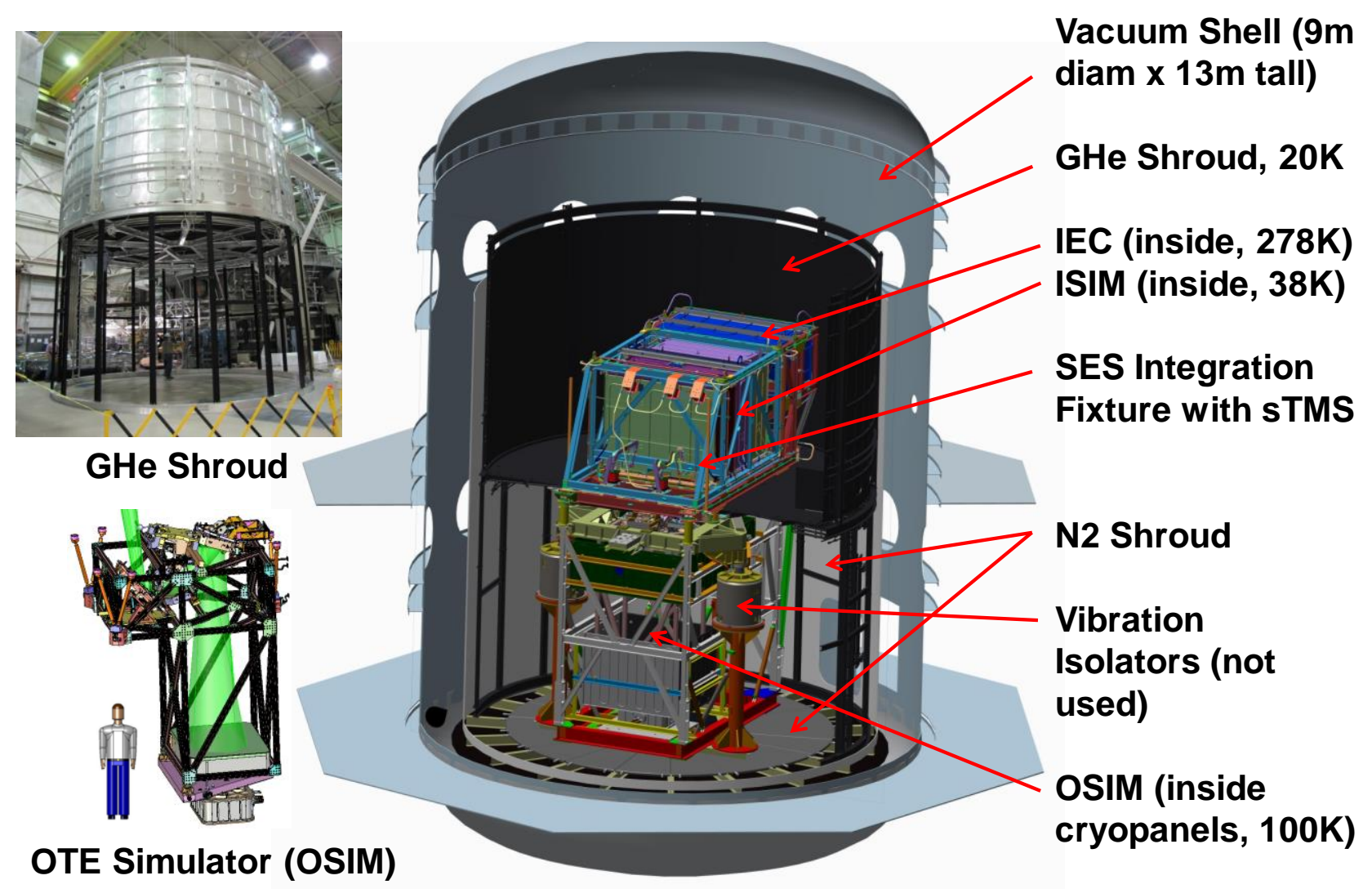

Figure 3. Right) Schematic diagram of the ISIM cryo-vac test configuration in Goddard's SES chamber. Representative temperatures are indicated. The sTMS (surrogate Thermal Management System) is the suite of cryopanels surrounding the ISIM Prime region, controlled to emulate the environment provided by the actual observatory Thermal Management System in flight. Upper Left) Photograph of the custom gaseous He-cooled shroud used for ISIM testing. Lower Left) Schematic of the OSIM telescope simulator. OSIM's fiber-fed or pinhole sources shine through holes in the first fold mirror at the upper right of its layout. They are then reflected off the spherical primary mirror at the bottom, back to the first mirror (with its small holes for the source inputs), off a second fold mirror, through the movable pupil stop (with a variety of selectable pupil elements), then off a final tiltable fold mirror upwards into the instruments.

A spacecraft simulator was mounted outside the chamber, providing power and commands to, and receiving data from, the ISIM hardware. The ISIM Command and Data Handling unit (ICDH), to be located ultimately in the JWST warm spacecraft, was also mounted outside the chamber - it had already undergone a separate thermal-vacuum test program. Additional cooling for the MIRI was provided through cooling lines from a GSE mechanical cooler mounted outside the chamber, but the ISIM-mounted cold head (the Heat Exchanger Stage Assembly) and plumbing were flight items.

\subsection{The as-built test configuration}

Photographs of the test configuration as actually implemented are shown in Figures 4 and 5. None of the flight hardware or even the OSIM simulator is visible, as these items are buried within cryo-panels and thermal blankets for thermal and stray light control. 
Figure 4. The "upstairs" configuration of the ISIM CV3 test - i.e. the region within the He-cooled shroud. The sTMS panels (and surrounding blanketing) are seen toward the right of the diagram; the IEC within its separate cooling panels is at the far right rear.

In the left foreground are the flight heat straps and the precision Q-meters that simulate the temperature of the flight radiator interfaces and accurately measure the heat flow from the instruments toward those radiators.

The large structure, whose beams can be seen, is called the SES Integration Fixture. Use of that assembly allowed much of the test configuration to be assembled in the clean room, and the entire assembly was then craned into the vacuum chamber for final closeouts and thermal and electrical hookups.

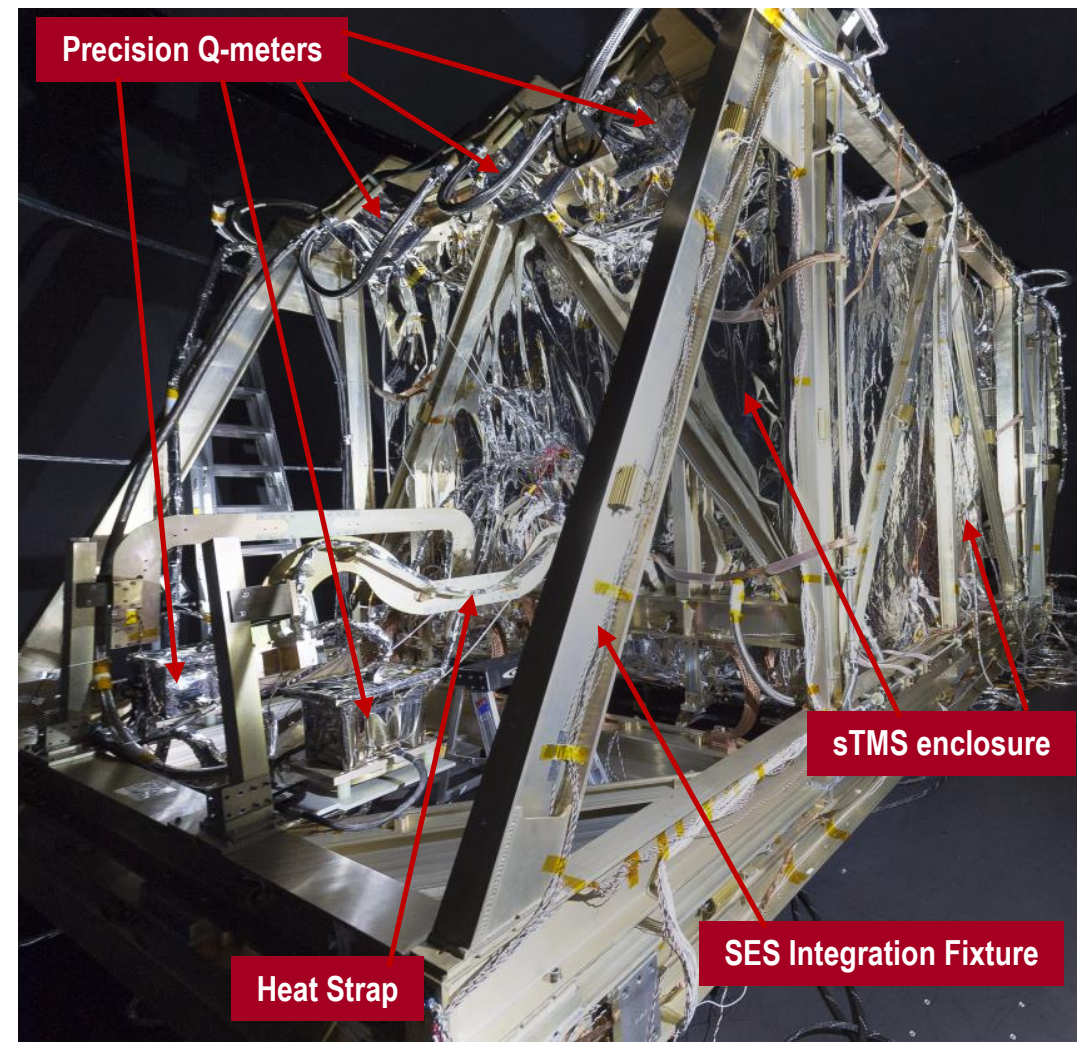

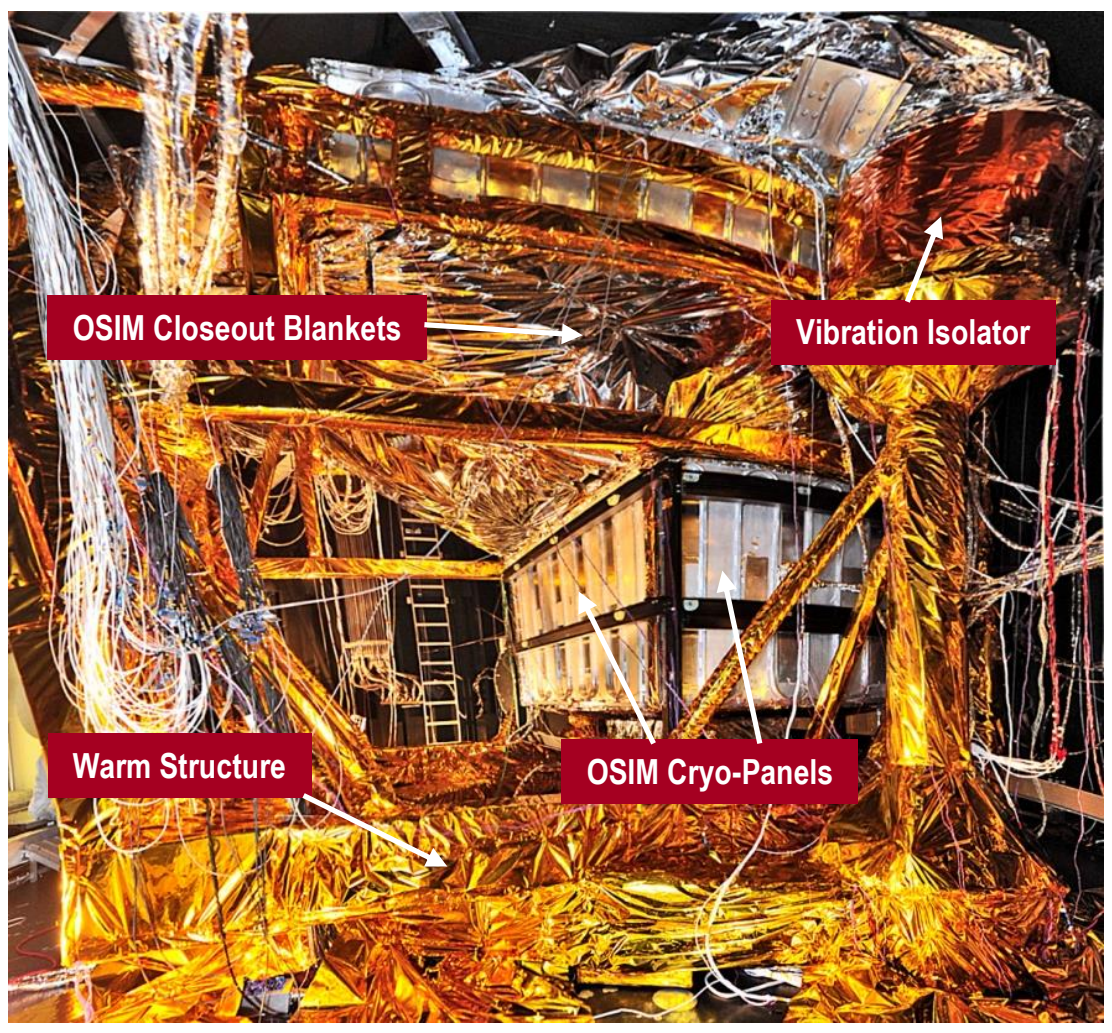

Figure 5. The "downstairs" region of the CV3 test, in which the OSIM telescope simulator was mounted, within LN2-cooled panels.

A triangular structure was mounted to the tops of the three vibration isolators. The OSIM hangs from that structure, which also supports the ISIM payload in the upper chamber, thus maintaining a rigid alignment between those elements. The pneumatic isolators did not need to be pressurized for ISIM testing; they provided passive support.

Many of the structures downstairs were held near room temperature, so the OSIM was carefully closed out with thermal blankets to keep that warm radiation out of the optical test environment. The $100 \mathrm{~K}$ operating temperature of the OSIM itself yielded acceptably low backgrounds in the nearIR instruments and in the shortest wavelength filter (F560W: 5.0-6.2 $\mu \mathrm{m}$ ) of the MIRI. 


\subsection{Personnel support}

During the CV3 campaign, round-the-clock support was provided by a substantial team. At least three chamber operators were on duty at all times; they and at least one thermal engineer (two during thermal transitions) were stationed just outside the chamber. The rest of the team was located in the ISIM Control Center within the same building complex. Staff there included at least a Test Director, Test Conductor, Test Operator (who issued the actual commands to the hardware) and Quality Assurance engineer. When instruments were powered or in thermal transition, there was at least one, and usually two, support persons from each SI team, along with two members of the ISIM optics team during optical testing and one or two members of the OSS team during OSS script testing. Additional personnel provided Ground Systems support and operational support for the MIRI GSE cooler. In all, there were up to 20 people on shift at any given time. In addition, numerous off-line team members provided test planning and data analysis support.

\section{CV3 TEST EXECUTION}

\subsection{Schematic of the CV3 test timeline}

A schematic overview of the CV3 test campaign as executed is shown in Figure 6. This rich diagram contains a wealth of information; the high-resolution image can be expanded in on-line viewing to make the details legible. We discuss the principal aspects of the test flow below.

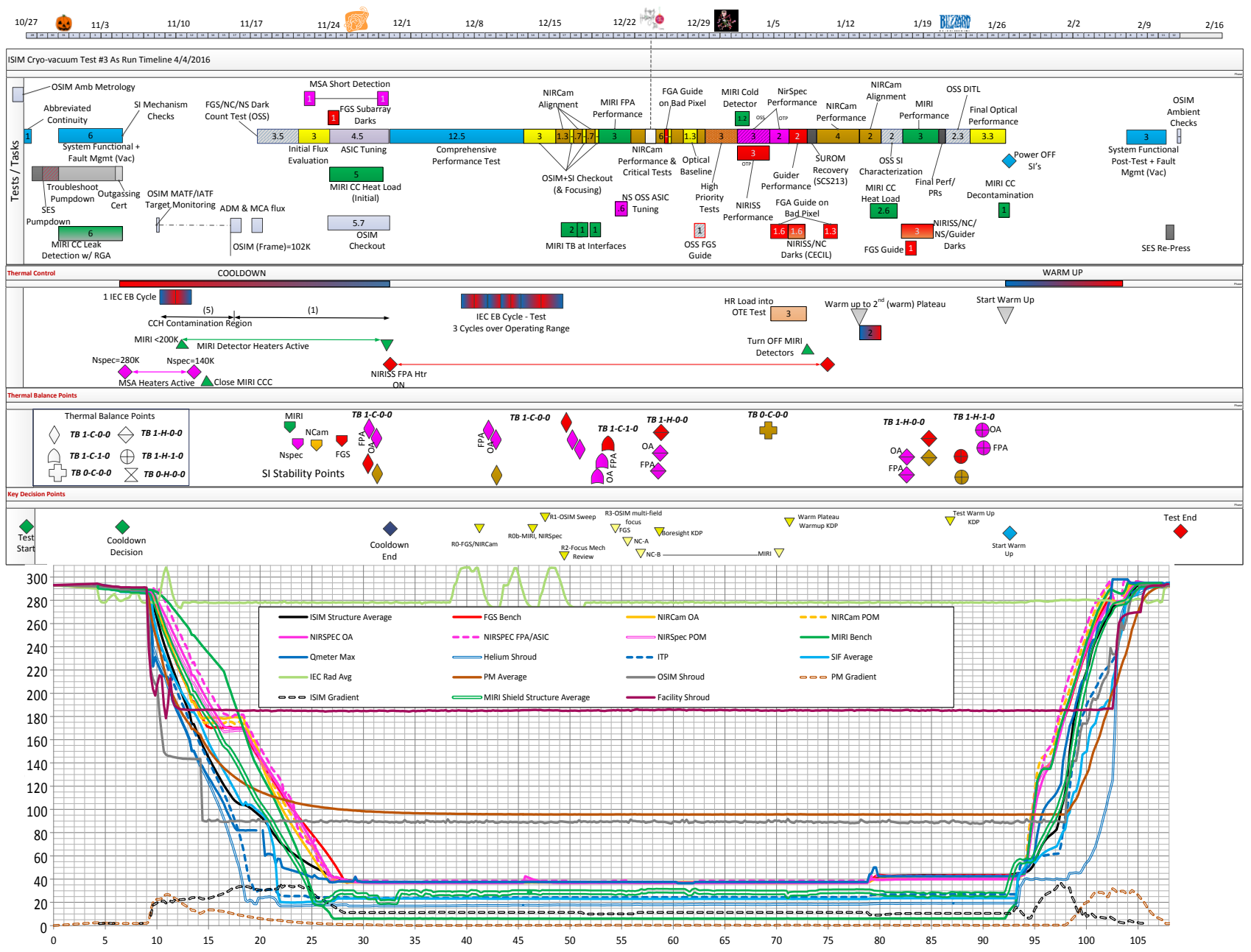

Figure 6. A schematic summary of the entire CV3 test campaign. The dates of the activities (month/day) are shown along the top. The upper portion of the diagram summarizes test activities, thermal phases, thermal balance points, and key decision points, while the lower panel of the diagram presents the temperatures vs. time for key system components. Zoom in on the figure to read the details of any items of interest. The various test phases and activities are summarized below. 
The CV3 test ran from an initial in-chamber electrical checkout and the start of pumpdown on October 27, 2015 through the backfill and opening of the chamber door on February 12, 2016, representing 108 days of round-the-clock operations through weekends, holidays, and a notable blizzard.

\subsection{Chronological overview of the CV3 campaign}

In the sub-sections below, we highlight the principal test activities accomplished in the various phases of the CV3 campaign. The key results of those activities are then summarized in the next section.

\subsubsection{Pumpdown and ambient temperature vacuum operations (days 0-9)}

The test began with an Abbreviated Functional and Continuity test of the hardware in the chamber; this brief electrical checkout verified that all connectors that had been de-mated in moving to the test facility had been re-mated. After successful completion of this test, pumpdown of the chamber commenced. Two days were lost initially dealing with leaks in the chamber; however, after this inauspicious beginning, the vacuum and cooling facilities performed superbly for the remainder of the lengthy test campaign.

Once high vacuum was successfully achieved, a System Functional Test was carried out, which exercised as much of the flight hardware as could be operated at room temperature, confirming more thoroughly the electrical connectivity of the setup and verifying the health of the hardware as much as possible before the decision to cool the payload was made. At the end of this ambient vacuum period, measurements of the condensable outgassing rate of the ISIM payload were made, which met requirements. At this point, the team caucused and agreed that the system could proceed to cooldown.

\subsubsection{Cooldown (days 9-28)}

The cooldown of the payload and GSE to the cryogenic temperatures was a slow and painstaking process. Numerous constraints on temperatures, rates of temperature change, and temperature gradients across structures had to be satisfied. In addition, temperature deltas among the various flight and GSE elements had to be monitored and properly maintained from a contamination standpoint, so that condensation of outgassing products did not occur onto critical flight systems. The relative temperatures of the flight instruments were controlled as they will be in flight (though with manual heater setpoint adjustments rather than the automated script control that will be employed in the telescope-level test and flight) - this process used the Contamination Control Heaters on the near-IR instruments to maintain their temperature at $165 \mathrm{~K}$ (above water vapor condensing temperature at the relevant pressures) until the ISIM composite structure dropped below $140 \mathrm{~K}$, and then stepped the SIs down together (along with the cryo-cooler controlled MIRI) in 5K increments until they all were below water-releasing temperatures, at which point their cooling could proceed passively, while using heaters to satisfy any remaining constraints.

The slowest element in the system was actually the OSIM, whose zerodur primary mirror could not be cooled excessively fast without developing structurally hazardous gradients. The instruments took nearly as long to reach temperature, however, arriving about 19 days after the start of the cooldown.

Many activities were able to proceed during the cooldown, however. OSIM alignment was tracked with its Alignment Diagnostic Module. The separately controlled IEC (which remained near room-temperature throughout, as in flight) underwent one of the four thermal cycles that it needed to complete its qualification program during this period.

In addition, many startup activities for the instruments were accomplished in the extended time period between 100K, when the near-IR SIs were powered on, and 38K, when the "cold operating" temperature setpoints had been achieved. These included: 1) the acquisition of warm darks for the near-IR detectors, useful as references for what to expect during in-flight commissioning and for assessing the state of the chamber stray light background; 2) a preliminary check of the health of the NIRSpec MicroShutter System; 3) execution of the Initial Flux Evaluation procedure. This latter activity exercised the OSIM pointing controls and the wide variety of OSIM light sources to establish pointing matrices and brightness settings for the many optical tests planned for later execution, enabling those tests to be executed with much less setup overhead during the cryo-stable testing period.

\subsection{3 “Cold-op” testing (days 29-78)}

Roughly four weeks after the chamber door was closed, the instruments and OSIM simulator achieved their target temperatures, and the cryo-stable phase of the test program could begin. A multitude of activities took place during this phase to achieve the verification and calibration objectives outlined above. We highlight here a few noteworthy aspects of the cryo-stable test flow. 
As previously noted, upgraded near-IR detectors, robust against hot pixel degradation, had been installed into three of the instruments between CV2 and CV3. For the NIRSpec instrument, the new focal planes came with new control and readout SIDECAR ASICs, to which the SCAs already had been electrically tuned in subsystem testing. In FGS/NIRISS and NIRCam, however, the SCAs were replaced, but the SIDECARs were not. Hence, an immediate priority was to carry out the electrical tuning process to tweak up performance of the SCA-ASIC combinations so that they were in a flight performance mode before the remaining calibration data of CV3 were obtained. This work was accomplished as soon as the SIs reached temperature, using the OSS system to loop over the relevant SIDECAR parameters; the data collected were analyzed expeditiously to select the optimum settings to be used for the rest of the campaign. Only small adjustments were made after this initial tuning period.

The so-called Comprehensive Performance Test (CPT) then collected verification and health trending data for detectors, mechanisms, calibration lamps, heaters, etc in all of the SIs, utilizing both sides of their and ISIM's redundant electrical systems. With all components of the SIs now available for operation (as opposed to during the earlier room temperature functional test), the CPT confirmed that the various systems had in fact survived the ambient environmental test program with good performance. Illuminated exposures in the CPT were executed exclusively with internal sources, i.e. with flight calibration sources mounted within the instruments themselves.

Test operations moved next to optical performance with external sources, using the versatile capabilities of the OSIM. The focus mechanisms in the near-IR instruments had specifically not been moved since the end of CV2, so that the stability of the alignment and wavefront error across the vibration and acoustic tests could be assessed. After that assessment was made, using the pointing and focus-scanning capability of the OSIM, then combinations of focus scans with the SI focus mechanisms and with the OSIM source mechanisms, over various points in the field of each SI channel, were used to determine optimized focus settings for each instrument. (Note that the MIRI, with greater depth of focus at its longer wavelengths of operation, does not have an internal focus mechanism - its fixed focus was simply determined to be within requirements.) Rapid analysis of the acquired data, along with close interaction between the SI teams and the ISIM optics team, permitted all of the focus decisions to be agreed upon expeditiously.

With detectors tuned and the instruments at best focus, the rest of the cryo-stable test campaign then could proceed. A variety of activities obtained the ISIM and instrument-level verification and calibration data outlined above. Meanwhile, thermal balance data was acquired for each of the instruments in various conditions (i.e. power on and off; with and without trim heaters powered), heat loads were measured into the MIRI cooler, the remaining qualification cycles for the IEC warm electronics were executed, etc. As Figure 6 indicates, there were often several activities running in parallel. The Lead Test Director, Begoña Vila, skillfully juggled activities as needed to keep the test moving efficiently, and the various teams showed great flexibility in responding to real-time deviations from the originally planned flow.

\subsection{4 "Hot-op" testing (days 79-93)}

After approximately seven weeks of testing at the cold operating temperature plateau, the temperatures of the sTMS environment and Q-meter sinks were raised to the hot operating plateau, for the acquisition of thermal balance data under those conditions. Key activities in this phase included MIRI testing (the MIRI, with its active cryo-cooler system, maintains a constant operating temperature over the range of observatory thermal environments, while the passivelycooled near-IR SIs do vary by a few degrees, though their focal planes have the option for active, closed-loop thermal control). The Onboard Script System was exercised extensively in this phase, with formal certification runs for various scripts as well as a "Day-in-the-Life" campaign intended to mimic the mix of activities that will be experienced in flight. A core subset of optical tests was repeated for trending, including a throughput cross-calibration for the near-IR SIs.

\subsubsection{Warmup and backfill (days 94-108)}

At the end of the cryo campaign, a formal decision meeting was held in which all stakeholders gave presentations confirming their readiness (sometimes pending the resolution of a small number of remaining test liens) to end the test campaign and start the warmup. Once those liens were resolved, the warmup began, again with the same highly controlled procedures to maintain the structural and contamination safety of the hardware. Two specific thermal activities were executed at the beginning of the warmup, one to assess the thermal response of the ISIM Prime region to a "decontamination" warmup of the MIRI cooling lines, a contingency operation in flight, and another to demonstrate that the Contamination Control Heaters in the ISIM were capable of raising the temperatures of the near-IR SIs to 165K with the surrounding environment still at cryogenic conditions, another potential contingency for flight. It took roughly two weeks for all hardware to reach room temperature; in the latter phases of the warmup, a final System Functional Test at vacuum was run to confirm the health of the hardware after this final ISIM-level cryo-cycle. CV3 was complete. 


\subsection{Invaluable software tools}

Before proceeding to an overview of CV3 results, we highlight some invaluable software tools developed by the team, without which time-efficient, safe execution of $\mathrm{CV} 3$ and the earlier ISIM tests would have been virtually impossible.

The first is the Test Planning Tool (TPT), an IDL GUI-driven tool developed for the efficient, configuration-controlled creation and execution of optical test scripts. ISIM testing involved four distinct SIs with at least a dozen different optical channels, leading to 18 separate detector arrays (with many different readout modes available), with dozens of filter, grating, and grism choices, and multiple available internal calibration lamps. For external illumination, these many channels could be fed by any of several dozen OSIM sources of adjustable brightness, temperature or timing (pulsed or continuous), pointed anywhere in the SI fields, with a variety of available pupil stops, and adjustable focus settings both within the OSIM and the near-IR SIs. It would have been absolutely impossible to keep track of all of the possible configurations for a given test exposure in any sort of manual way and to update these configurations safely and reliably as the test unfolded.

The TPT - conceived by Scott Antonille of the ISIM Optics Team and developed by a skilled team of software developers and data analysts under his leadership - provided a controlled way of 1) selecting a complete SI + OSIM exposure configuration, 2) predicting the position, morphology, and brightness of the resulting image, 3) checking the compliance of the configuration with allowed combinations and against engineering constraints and limitations, 4) assembling a sequence of such observation configurations into a complete test, 5) tabulating the use of limited life items, and 6) fully documenting the sequence in a set of conveniently reviewable outputs. The configuration list from the TPT was then fed, when released for execution, to a similarly impressive software script, "otp_execute", developed primarily by Raymond Wright of the ISIM Operations Team, which parsed the sequence of exposure configurations from the TPT into a valid sequence of ISIM, SI, and OSIM commands to execute those exposures, building in all appropriate rules of sequencing of mechanism, lamp, detector, and OSIM operations, as well as managing the data output paths. A strong advantage of the TPT is that it is structured so that updated information about OSIM pointing or the brightness of a light source can be input so as to propagate automatically to all other planned tests using that pointing or source. See Antonille et al. (2016) ${ }^{15}$ for a more detailed description of these impressive software tools.

Regarding thermal operations, we have previously noted the complexity of executing cryogenic operations (especially the thermal transitions) while satisfying the numerous structural and contamination-related constraints. To aid the thermal engineers in this process, another impressive software package was developed, primarily by Brian Comber of the ISIM Thermal Team. This software system (utilizing Microsoft Excel and Visual Basic) was called Fusion ${ }^{12}$, because it fused the output of the two different thermal data streams (the flight telemetry reporting on flight thermal sensors, and an existing facility test system, reading out the GSE sensors), and provided a single monitoring system for the roughly 1700 (!) total sensors in CV3. While the two underlying telemetry systems provided the usual ability to monitor against yellow and red limit values on individual thermal parameters, the Fusion system in addition enabled any programmed combination of parameters to be evaluated for any approaching violation of rates, spatial gradients, or temperature differences between items. In addition, the Fusion system leverages the capability of Excel to do conditional formatting to indicate alarm status (both visual and audible), to create graphs commanded by the syntax, etc. The system eventually grew to recommend mitigations in the event of approaching limit violations, or what procedural steps to do next (i.e. during cooldown or warmup) when certain conditions were met, to the thermal engineer on duty. These capabilities proved invaluable in the safe and efficient execution of the complex cryo-operations of CV3.

\section{OVERVIEW OF CV3 RESULTS}

\section{All major objectives of the CV3 test campaign were accomplished. The hardware performance was excellent, and the instruments and ISIM systems were shown to be in excellent health after the environmental test program.}

The single exception regarding the health of the hardware was an issue that was already known from ambient functional testing in the clean room before CV3: a redundant detector thermal control board (located in the warm IEC) for one of the MIRI channels showed intermittent erratic behavior. It was decided to proceed to CV3 while the behavior was investigated. CV3 operations confirmed the faulty behavior of that circuit. As planned, the full suite of MIRI CV3 test objectives were accomplished nonetheless using the primary side electronics board. The origins of the fault were identified. Once CV3 was over, the offending electronics were removed from the IEC, and the fault was confirmed; the electronics were repaired, tested at the subsystem level, and re-installed into the IEC, which is now ready to proceed to integration with the telescope. The cryo-performance of the repaired circuit will be confirmed in next year's telescope- 
level cryo-vacuum test, which will take place after the telescope-level ambient environmental test program; the reworked IEC will thus see all appropriate test environments and a final cryo-test before launch. NO hardware deficiencies requiring rework within the cold portions of the SIs were identified in CV3. Those regions remain undisturbed.

The results of CV3 have been thoroughly vetted in a number of formal reviews of optical performance, thermal performance, and the performance of the SIs. The results of those reviews then formed a key part of the official ISIM Pre-Ship Review that documented the readiness of the ISIM to proceed to integration with the telescope. "Shipping" in this case consisted of moving from one side of the GSFC clean room to the other, to meet up with the flight telescope. CV3 results also form the backbone of many of the Requirements Verifications Reports that the ISIM Systems Engineering team is currently completing to formally document the compliance of the ISIM science instrument suite with its numerous requirements. Also, from CV3 data, the SI teams are preparing the calibration references files (darks, flats, linearity, etc) that will underpin the science data processing pipeline for analyzing observations in flight.

In the sub-sections below, we highlight some of the noteworthy results of CV3.

\subsection{Optical results - alignment}

A critical objective of the CV3 campaign was to confirm the six degree-of-freedom alignment of the instrument channels to the simulated OTE focal plane and exit pupil provided by the carefully validated OSIM telescope simulator. This aspect of CV3 was highly successful, as reported in detail by Rohrbach et al. (2016) ${ }^{16}$. The alignments were shown to be very stable through vibration (through comparison to similar results from CV2, confirming as well the success of the meticulous metrology executed during the SI upgrades) and to meet the key requirements. Those alignment results are summarized graphically in Figures 7 and 8, provided by Joseph Howard of the ISIM Optics Team.
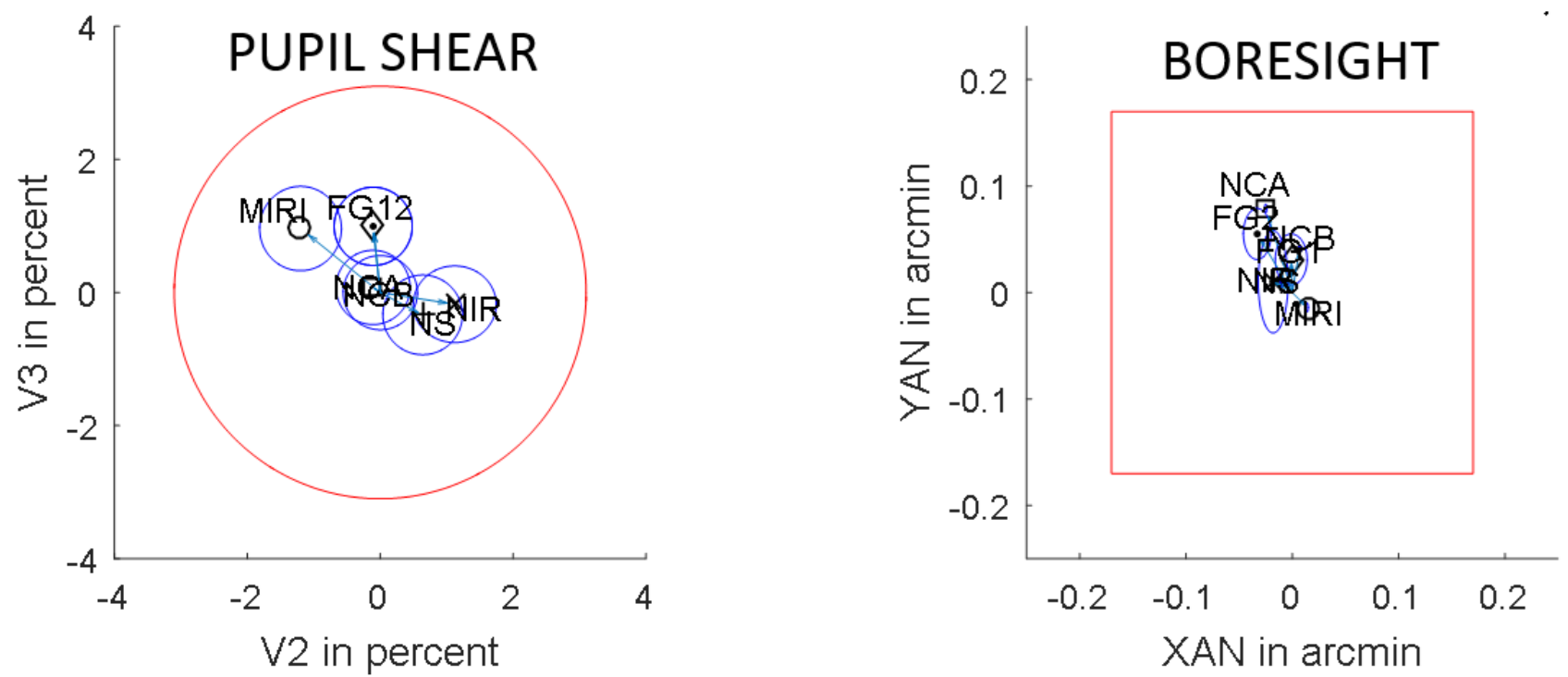

Figure 7. Alignment results for the various channels of the SIs. The results for each channel, along with the two- $\sigma$ error ellipses, are shown in blue, and the ISIM-level allocation is shown in red. Left) Pupil Shear of the SI pupil vs. the telescope pupil, measured in \% of pupil diameter. Right) Instrument boresight center in arcminutes on the sky vs. nominal.

Figure 7 demonstrates that the pupil alignment of the SIs, a critical parameter for this open-architecture IR telescope, is well within the ISIM allocations for all channels - misaligned pupils could allow light into the SIs from unwanted paths. (Note that in the raw data, the NIRISS channel pupil shear was closer to the limit, though still within allocations - the value plotted here assumes a planned software update to the nominal rotational position of the NIRISS pupil wheel, which will reduce the pupil shear in the tangential direction of mechanism rotation without introducing any vignetting.) The Boresight alignment of the SIs is also seen to be well within allocations.

In the FOCUS portion of Figure 8, it is assumed that the near-IR SI focus mechanisms have been driven to best focus, to within the measurement uncertainties determined in CV3. The important things to note about this are the following: 1) MIRI, with no internal focus mechanism, is well within requirements in its fixed position; 2) the two Guider channels, 
which share a common focus mechanism, are sufficiently parfocal that the "compromise" position for their mechanism leaves both Guider channels with negligible focus offsets; 3 ) the variations over the field and from one filter to another within the SIs are within requirements; and, finally 4) the near-IR focus mechanisms have sufficient remaining range to cover the required allocations for the higher levels of assembly of the instruments into the observatory.
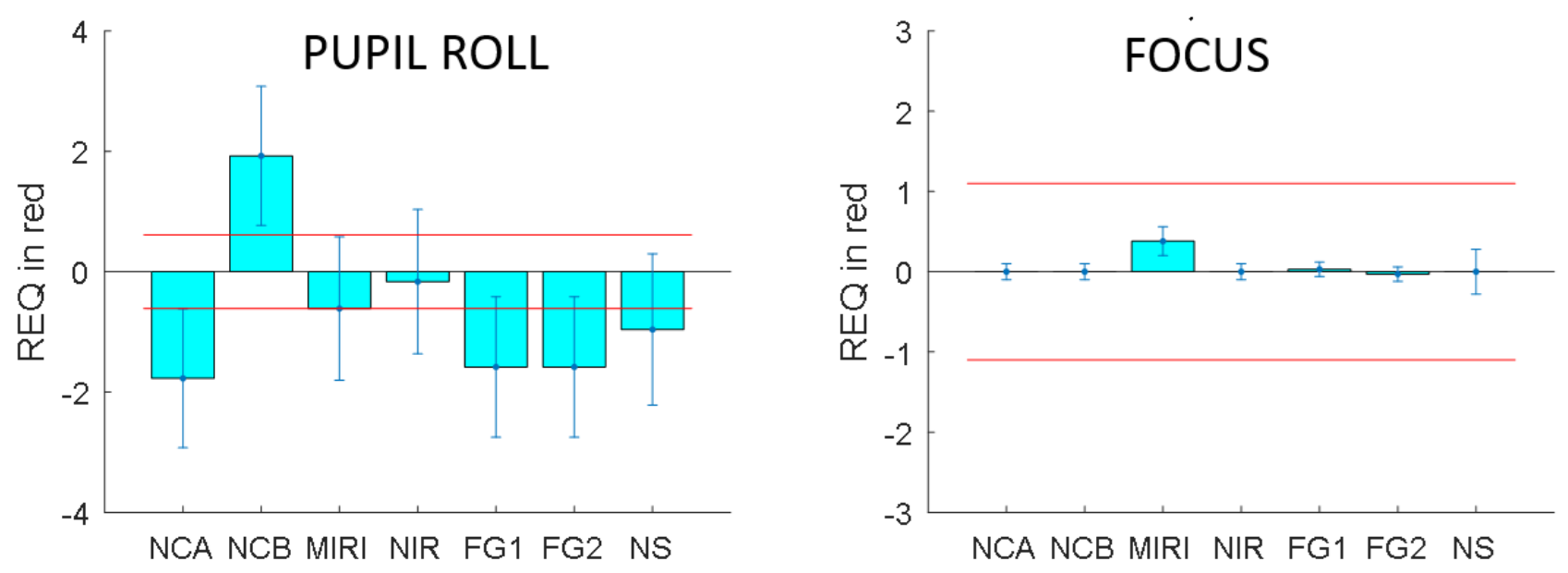

Figure 8. Alignment results for the various channels of the SIs. The results for each channel, along with the two- $\sigma$ error bars, are shown in blue, and the ISIM-level allocation is shown in red. Left) Pupil Roll of the SI pupil vs. the telescope pupil, measured in degrees. Right) Focus of the instruments, after the near-IR instrument focus mechanisms have been driven to best focus within measurement uncertainties.

Pupil Roll stands out as the one area where the baseline requirements were not met (or in some cases where the error bars extend beyond the allocations). This was seen in CV2, and the results were quite stable between CV2 and CV3. When the CV2 results were obtained, a great deal of analysis was done to see if there are in fact any serious deleterious consequences to this degree of roll misalignment, as it was clear (e.g. from the equal and opposite misalignments of the two NIRCam modules) that no global roll of the ISIM module on its kinematic mounts could eliminate them. It was found that the pupil roll requirements had been made unnecessarily tight, and the level measured in CV2 and CV3 is in fact acceptable. In particular, it was specifically demonstrated that cases where the pupil alignment does seriously matter, e.g. in keeping the NIRCam Dispersed Hartmann Sensor elements aligned with the Primary Mirror segments that they are supposed to uniquely sample, the measured performance was within the necessary range to yield the desired performance (see Section 4.5.3). Hence, no adjustments were made within the SIs between CV2 and CV3.

The upshot of all the six degree-of-freedom alignment measurements was that no adjustments to the kinematic mounts that mount the ISIM to the OTE were required; that installation has since taken place, and optical metrology indicates that all alignment requirements of the installed ISIM (except for the pupil roll requirements to be waived) are met.

\subsection{Optical results - wavefront error}

Another critical aspect of the optical performance evaluated in CV3 is wavefront error (WFE). In addition to reverifying the SI-level WFE requirements for channels that were disturbed during the pre-CV3 upgrades, it is essential from an observatory commissioning standpoint that the WFE of the SIs (most particularly the NIRCam) be both well calibrated and demonstrated stable before launch. Optimization of the telescope alignment during commissioning demands that wavefront errors due to misalignments of deployed/adjustable optics (the Primary Mirror Segments, the Secondary Mirror) not be confused with WFE intrinsic to the SI channels.

Hence, a major portion of the optical test program involved focus sweeps to acquire the data for image-based wavefront sensing (or "phase retrieval") analysis. In both CV2 and CV3, the appropriate data sets were successfully acquired and enabled a thorough analysis for all SI channels. We do not elaborate on the details here, which are presented thoroughly in this volume by Aronstein et al. (2016) ${ }^{17}$. We simply note that overall, both the WFE performance and the accuracy of its determination were excellent. Figure 9 presents an example of the sort of focus sweep data acquired and the wavefront error map derived as a result. 


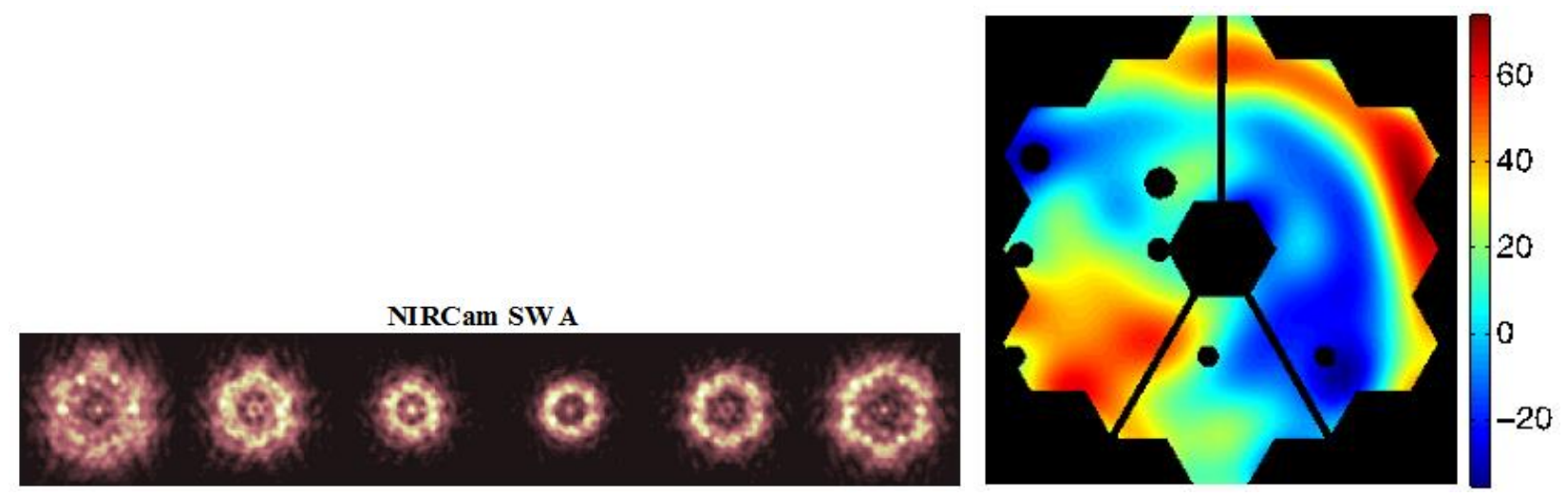

Figure 9. Left) Sample images from a focus sweep through the ShortWave channel of NIRCam Module A, at a wavelength of $2.12 \mu \mathrm{m}$. Right) The resulting low-frequency (5 cycles per aperture) WFE map derived from the data. (Mid- and hifrequency WFE were also assessed.) The irregular "tricontagon" periphery and the central obscuration and spider legs are flight-like pupil features, representing the edges of the JWST segmented primary and the Secondary Mirror Support Structure. The dark holes are data missing from the pupil map due to the presence of holes in the OSIM simulator's first fold mirror, through which the input illumination is injected.

\subsection{Thermal results}

The thermal objectives of CV3 were successfully accomplished. First and foremost, the hardware was managed safely through the cooldown, cryo-operations, and warmup. There were a few small limit violations, but none that raised any serious concerns. Noteworthy thermal results of the campaign include the following (see Glazer and Comber [2016] ${ }^{12}$ for further details):

- The SI and ISIM temperatures, the heat loads from the SIs to their simulated radiator sinks, and the heat loads from the Harness Radiator to the ISIM, were measured accurately at the cold and hot operating temperatures. Multiple thermal balances were obtained, with the SIs powered On and Off, and with their Trim Heaters powered On and Off. The data obtained fully support the critical CV3 objective of validating the ISIM thermal model for use within the broader Observatory thermal model for verifying those ISIM requirements that must be verified by analysis.

- The workmanship of the flight thermal elements was confirmed. The high-conductivity heat straps, all of the temperature sensors that can be tested at ISIM level, the primary and redundant heaters are all working properly.

- Power dissipations by the SIs were measured to better than $2 \mathrm{~mW}$ uncertainty using precision Q-meters (see Glazer and Comber [2016] $]^{12}$ and references therein for detailed information about the design and calibration of these remarkably accurate devices). All were in good agreement with SI-level test data, all agree well with CV2 measurements, and all meet ISIM requirements.

- The thermal conductances of the high-conductivity heat straps and their interfaces were measured and were satisfactory. This was a very significant result, as measurements after CV2 had indicated significant issues with creep and loss of pre-load at joints between the heat straps and the structures they mount to. An extensive test program before CV3 validated modified designs and torquing methods for those interfaces. The CV3 results confirmed that the modified interfaces maintained their preload through the ambient environmental test program and when cooled for flight-like operations.

- The heat load into the MIRI cryocooler was measured under several conditions and also met requirements.

- The total heat load from the Harness Radiator into the SIs and into the OTE also met requirements.

- The upshot of all of the measurements in CV3 is that the ISIM system is comfortably cold-biased, as is essential for this passively cooled (except for MIRI, with its mechanical cryocooler) instrument suite. There is adequate heater power available to maintain SI temperatures over the range of uncertainties in the flight environmental predictions.

- Useful data were collected to characterize the response of the ISIM to two off-nominal operations: annealing of the NIRSpec MicroShutter arrays, and decontamination warmup of the MIRI cryocooler lines. 


\subsection{Operations results}

CV3 provided (as did the previous ISIM cryo-tests) an invaluable opportunity to gain operational experience with the SIs and ISIM systems. Some of this involves formal certification of software and scripts; some is simply accumulating runtime to gain familiarity with ISIM operations: exercising command and data paths, gaining experience with data pipelines, learning which telemetry monitoring limits need to be modified, exercising Fault Management paths, etc.

Some specific operations outcomes included: 1) certifying $124(\sim 45 \%)$ of the Flight Realtime Command Procedures ("CECIL scripts"; CECIL stands for C-Extended Command Interface Language) against the flight hardware (the remainder will be certified by the end of the telescope-level cryo-test next year); 2) verifying the successful fixes to previously identified flight software issues (e.g. interrupt handling problems in the data output paths that had led to data overwrites and even system crashes during CV2); 3) gaining significant run-time with various Telemetry Monitors (TMONs) enabled; 4) flowing >25,000 images and 8 terabytes of raw data through the system.

\subsubsection{The Operations Scripts Subsystem}

An area of particular interest in operational testing was the use of the Operations Scripts Subsystem (OSS), whose development is led by the Space Telescope Science Institute (STScI). OSS provides the onboard scripts containing the science instrument commanding activities, written in JavaScript, and implements the Event-Driven Operations paradigm for JWST. The high-level SI activities are launched from "visit files" in the order listed in an "observation plan" and executed by the Observation Plan Executive (OPE), all of which are resident onboard.

The main goal for OSS during CV3 was to certify most of the 720 onboard scripts for flight and all of the OSS script features against the hardware in a flight-like environment. OSS provided end-to-end testing for the ground and flight system. In addition to these direct goals of OSS certification, OSS was a valuable tool for carrying out activities in support of other objectives in the CV3 flow, providing greater efficiency (especially when other non-OSS activities could be run in parallel) in addition to providing general experience with OSS use.

Early CV3 examples of OSS-led activities include the acquisition of SI detector "warm darks" during the cooldown period and the execution of the SIDECAR ASIC tuning process for NIRCam, FGS, NIRISS, and NIRSpec (a brief proof-of-concept of the tuning process was executed for NIRSpec even though that SIs new SCAs came in pre-tuned with new ASICs); the tuning exercise both acquired the data needed for realtime optimization of the SCA-ASIC performance for the remainder of CV3 operations and validated the process for in-flight tuning should that ever be required. OSS generated a grand total of 3729 exposures for the SI teams to tune their detectors over a period of 5 days.

OSS also was utilized extensively in tests of the critical Guiding function with the FGS. Early on, in preparation for other OSS-led tests later in CV3, OSS performed a 10 hour FGS Guiding Checkout test. OSS successfully identified the target brightness and location of 10 simulated guide star sources (from the OSIM) with FGS1 and FGS2; several test cases had offsets from their nominal positions. Then, OSS repeated the process, going all the way through the various phases of Guiding from ID and Acquisition, through Track, to Fine Guide. The resultant simulated guide star locations were used later in the OSS SI Characterization Test and the OSS Day-in-the-Life Test when guide stars were needed.

In the NIRSpec Performance Test, OSS provided 3.5 days of continuous internal calibration exposures, configuring the MicroShutter Array (MSA) and flashing internal calibration lamps, in various spectral modes, in support of the development of the revised optical model of NIRSpec required by the pre-CV3 installation of new MSA and detector hardware. While this OSS-led activity was ongoing, NIRISS performance characterization tests were run in parallel using non-OSS scripts, saving valuable test time.

Two major OSS activities were carried out during the "hot op" period of the cryo-test program: the SI Characterization Test (SIC) and the Day-in-the-Life Test (DIL). The two-day SIC test commenced by reconfiguring each science instrument down and back up and then by executing a total of 177 visits testing each science instrument's OSS features. Twelve new OSS features were verified, 15 more OSS Level 4 requirements passed, and 563 OSS scripts were certified to Level 3 status against the instrument hardware. In addition, the Spacecraft feature scripts were integrated into the OSS script set and were exercised against the Spacecraft 2A Simulator successfully.

The last OSS led test was the DIL, which ran for two days during the U.S. East Coast Blizzard of 2016. The DIL visits for this test were generated from 26 proposals provided by STScI scientists using the JWST Astronomer Proposal Tool (APT). Each proposal was processed through the Visit Scheduling System (VSS) and the Observation Plan Generation System (OPGS). The DIL visits executed flawlessly and generated 1666 exposures among all of the science 
instruments. Unlike the other OSS tests run during CV3, the EM-SSR (Engineering Model Solid State Recorder) was used, and the science data dumps were managed in a flight-like manner (most CV3 tests flowed data through an alternate path that is more efficient for ground I\&T). This test provided an "end-to-end" test of the JWST ground system from proposal to DMS and was successful in demonstrating its capabilities.

OSS performed well during CV3, validating its scripts and features, providing useful science data to ISIM I\&T, finding new issues, and running in a flight-like way.

\subsection{SI results}

Thus far, we have emphasized the results of CV3 primarily at an "ISIM level", regarding global optical alignment, thermal performance, and operations. Numerous CV3 activities also obtained data relevant to "instrument level" health, performance, and calibration. Some of those results are presented elsewhere in this volume ${ }^{4,18-20}$. Below, we briefly note some highlights of the numerous, varied SI test results that were obtained in CV3, as presented by the SI teams during the JWST Project's post-CV3 data reviews. This brief section is of course not intended to formally or comprehensively document the scientific capabilities of the SIs on board the observatory. The Call for Proposals for JWST Cycle 1 observations, to be issued in late 2017, along with its supporting documentation, will serve as the official resources for the astronomical community with respect to the expected performance of JWST and these SIs in flight.

\subsubsection{FGS/NIRISS}

FGS results are presented in this volume by Rowlands et al. (2016) ${ }^{18}$. As previously noted, tuning of the new SCAs to the ASICs was a key test objective for the FGS and NIRISS; this was accomplished through the acquisition of data while looping over four different adjustable ASIC parameters, for a total of 192 configurations in the initial run. CDS noise, Intra-Pixel Capacitance, PSF symmetry, and crosstalk were key parameters assessed, along with (for NIRISS) total upthe-ramp noise (for the very short Guiding exposures, CDS noise is more important). The upshot was very satisfactory settings, compliant with requirements. With the optimal settings selected for the rest of CV3, calibration data were acquired to support the development of the flight data pipeline: darks, flat-fields, and linearity measurements. The latter two utilized the external flat-field capability provided by the OSIM diffuser, a late "best-effort" addition to the OSIM design, which proved invaluable when the post-SI-delivery installation of new detectors required that such calibration data be re-acquired during ISIM-level testing. The new SCAs also showed excellent cosmetics and pixel operability, with a low, stable population of hot pixels as expected for the improved SCA design.

Testing of the Guiding functionality was of course a major part of the FGS test campaign. Numerous test sequences went through the sequence of modes from ID (Identification) and Acquisition, through Track, to Fine Guide. Extended guiding runs were made using OSIM sources, while other SIs were operating, as will be the norm in flight. In addition, by unmasking a hot pixel, and setting up to guide on that bright source, very extended continuous guiding runs could be executed with no constraints on OSIM pointing, so that other optical test activities could proceed in parallel. These were not closed-loop activities - Guider error signals did not feed back into OSIM pointing - but the runs tested the operational aspects of the mode transitions and the ability to run continuously for long periods without error.

The fundamental Guiding metric - Noise Equivalent Angle (NEA, the rms magnitude of the error signal produced by the Guider for a nominally stationary source) - was assessed in a variety of tests with different source fluxes and spectral energy distribution. An example of the excellent NEA vs. source flux achieved with the new detector arrays is shown in Figure 10. The offsets between the centroids found in Track Mode vs. Fine Guide mode (the process is slightly different) were shown to agree within requirements, and the proper response to moving targets was also confirmed.

Another interesting set of guiding tests involved defocusing the OSIM input sources to represent the aberrated image shapes that will be presented to the Guider in the early stages of telescope commissioning, when the OTE optics are not yet aligned. The ability of the Guider to work with these non-stellar PSF's was demonstrated and necessary brightness levels established.

On the NIRISS side of the instrument, the replacement grisms were optimized for spectral tilt (by fine-tuning the rotational setpoints of the wheel on which they're mounted); a wavelength calibration was also performed at a few discrete OSIM wavelengths, in excellent agreement with the optical models. Performance (e.g. repeatability) of the refurbished Dual Wheel mechanism itself with the new motors (improved planetary gears) was also characterized.

Finally, demonstration observations were made with the Aperture Masking Interferometry mode (that uses the NonRedundant Mask in the Pupil Wheel) and in a high S/N time series with the GR700XD grism, as will be used for 
exoplanet transit spectroscopy. The latter observations have motivated the development of a new readout mode for the near-IR SCAs that will permit higher duty-cycle observing for such cases.

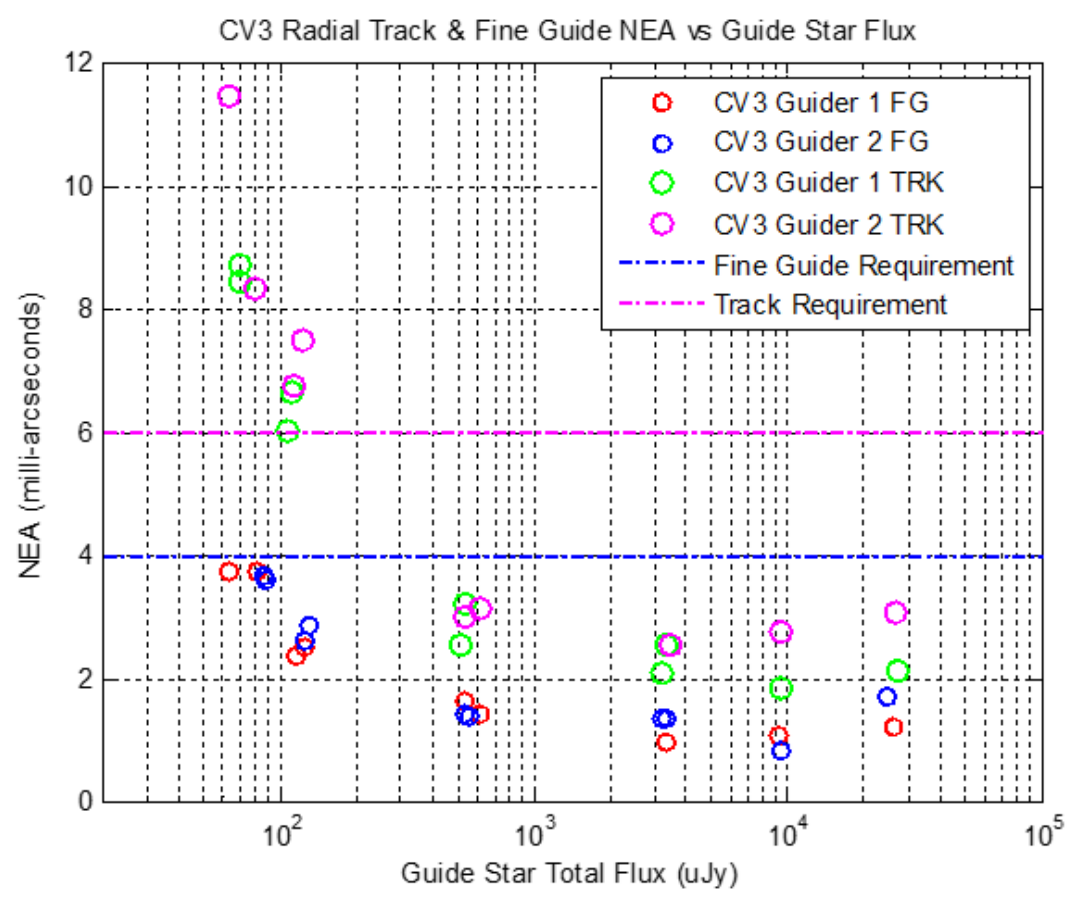

Figure 10. Guider NEA vs. equivalent guide star flux, as measured in CV3. The NEA declines to an asymptote rather than continuing to decline with increasing source S/N, due to a small amount of OSIM source jitter over the periods of Guider integration. Nonetheless, the data confirm that the measured NEA meets requirements. From Rowlands et al. $(2016)^{18}$.

\subsubsection{MIRI}

There have been no changes made within the MIRI Optical Assembly after delivery of the instrument (hence none between CV2 and CV3). Trending measurements for the mechanisms, lamps, imaging filters, and spectrometer elements showed excellent health, with nominal optical performance and stability throughout the ISIM environmental test program. Optical requirements were verified at SI-level and remain compliant in ISIM cross-checks.

However, improvements to the Focal Plane System electronics chain (on boards that reside in the warm IEC) were made before CV3; a number of CV3 measurements confirmed the good performance of those electronics and confirmed the optimal bias settings to be used, as had been identified in off-line testing with a detector testbed at the Jet Propulsion Laboratory. A systematic improvement in read noise performance in the SLOW mode of readout was observed in CV3. Another goal of the detector performance checks was to confirm the consistency of the results obtained under OSS control vs. results obtained with the lower-level CECIL commanding. Consistency was in fact demonstrated, providing good trending continuity between the ground cryo-test program and the corresponding commissioning activities that will be run with the OSS in flight.

The capabilities of the OSIM were used to test flight dither patterns and to make measurements of the slice losses in the Medium Resolution Spectrograph (MRS) IFU, demonstrating very tight constancy of the photometry, within $\pm 0.2 \%$. The MRS point spread function was also measured with the highest accuracy to date. Excellent flat fields were obtained (see Figure 11), including subarray flat fields in the new BURST readout modes that provide speed gains (higher dynamic range for bright sources) in small subarrays. The very uniform OSIM external flats are beneficial for learning how to optimally use MIRI's internal lamps for generating and tracking any time changes of pixel-to-pixel flats. OSIM point sources were also used to study long time constant latent images.

The problem seen before CV3 with one MRS channel's Side B detector thermal control board was investigated in CV3, including assessing how the detector performance would be affected should that problem not be resolved. Fortunately, those results were rendered moot by the successful analysis and repair of the board, as will be verified at JSC next year. 


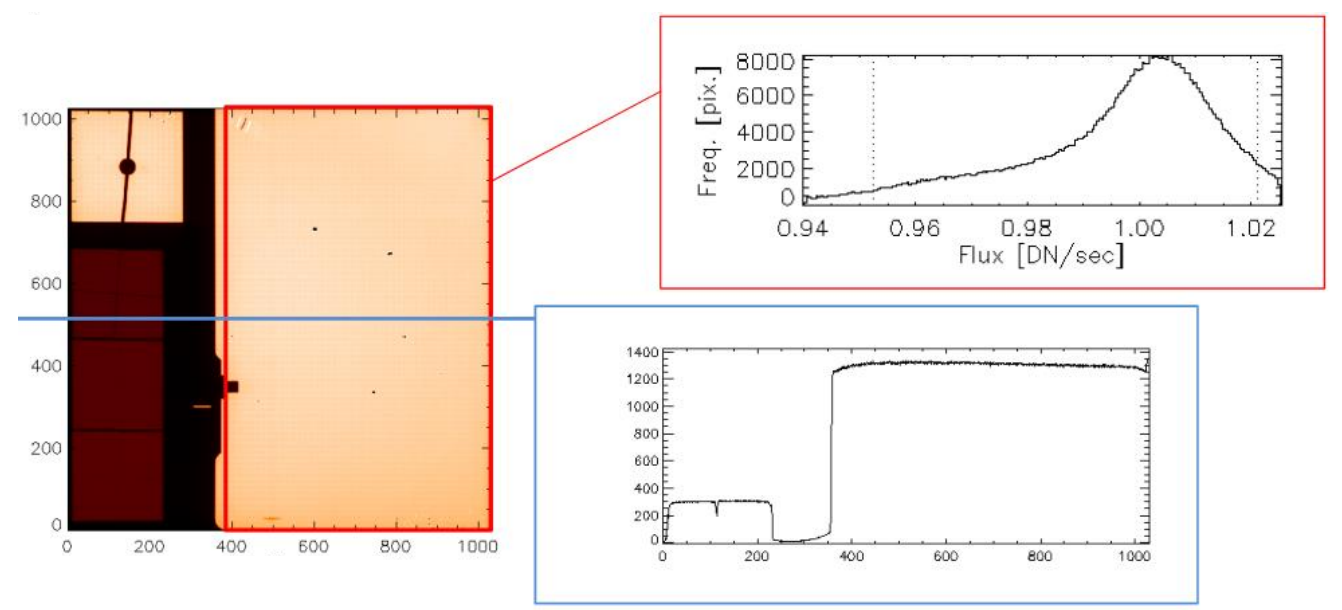

Figure 11. Sample of the excellent flat-field data (left) obtained for the MIRI Imager with the OSIM diffuser. The upper right plot shows a histogram of observed data values, with $96 \%$ of the pixels within a $4 \%$ response range. The lower right plot shows the smoothness of a cut along a detector row. Figure courtesy of Alistair Glasse and the MIRI team.

\subsubsection{NIRCam}

For NIRCam, ASIC tuning was required for the 3 new SCAs installed between CV2 and CV3, but the parallel readout of all 10 arrays during the tuning process allowed the tuning of the unchanged arrays to be revisited as well. 240 iterations, adjusting five ASIC parameters, were investigated in CV3 (a much-reduced set vs. the first tuning exercise in CV2). Metrics assessed included read noise, 1/f noise, Post-Pixel Capacitance (extra signal in a trailing pixel due to settling), noise stability, and power consumption (more of an issue for NIRCam with its 10 detectors).

Excellent results were obtained, satisfying noise requirements for all channels. Explicit tests verified that the noise performance is not affected by enabling the optional closed-loop detector thermal control, nor by observing simultaneously with the ShortWave and LongWave Channels in a module. With final tuning in hand, reference darks, flats, and linearity measurements were obtained, which will provide the calibration pipeline with bias files, bad pixel masks, pixel-to-pixel flats, gain, interpixel capacitance, linearity, and saturation level. No growth in hot pixels was seen from CV2 to CV3, again confirming the robustness of the new SCAs. One software update request resulting from the test (currently being worked in a prioritized queue) is offset control for individual readouts, which will improve the full well and thus the dynamic range for some of the SCAs whose four readout channels differ significantly in video offset.

Health checks included trending of NIRCam engineering telemetry; no concerns were noted. In addition, Point Spread Function profiles were measured through all filters and compared with theoretical profiles. No evidence of blue or red leaks was seen; nor were there signs of degradation between CV2 and CV3.

As previously noted, the excellent wavefront error results for NIRCam as well as the other SIs are presented in detail in Aronstein et al (2016) ${ }^{17}$. Other aspects related to the detector replacement accuracy that were successfully checked in CV3 include 1) confirming the sharpness of focus of the Coronagraphic Occulting Mask (COM) to the detector plane that is a fixed focus, not adjustable with the mechanisms that focus the overall NIRCam modules to the OTE; 2) confirming the required overlap of the ShortWave and LongWave Channel fields of view within each module; and 3) confirming minimal vignetting by the focal plane masks that were replaced before CV3.

A noteworthy aspect of NIRCam testing was a number of activities related to NIRCam's role as the primary wavefront sensor for commissioning of the observatory and for monitoring its wavefront throughout the mission. In support of these activities, requirement checks and calibrations were made related to, for example, NIRCam's Pupil Imaging Lens (PIL) and the Dispersed Hartmann Sensor (DHS) grisms. For the PIL, unvignetted field of view, pupil image distortion, and pupil image size were checked and confirmed to meet requirements. For the DHS, measurements confirmed the proper registration of the DHS segments with the primary mirror segments they are intended to measure (see Figure 12). In the LongWave Channel, the grisms - which offer a contingency method for the coarse phasing step of observatory commissioning - were adjusted to proper spectral tilt and measured for dispersion (and their utility for science use in exoplanet transit observing was also noted!). 


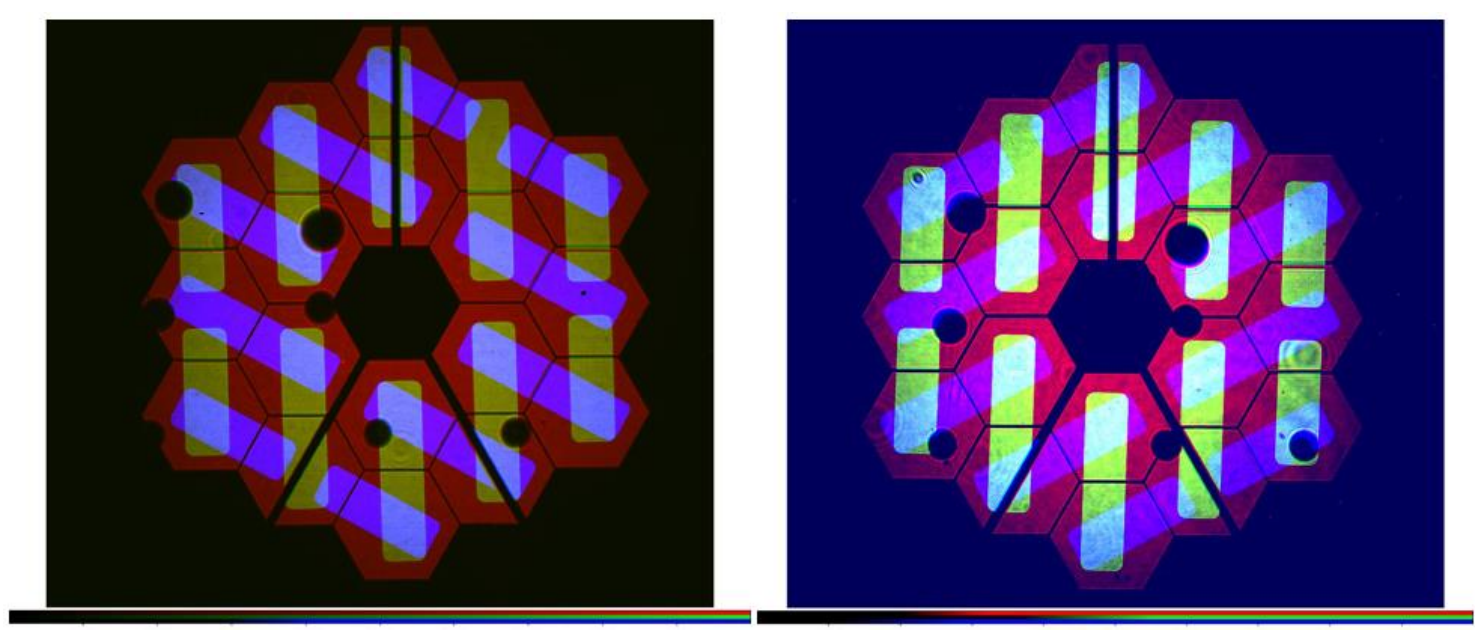

Figure 12. CV3 demonstration of the alignment of the NIRCam Dispersed Hartmann Sensor (DHS) grisms to the nominal OTE pupil. Each image (one for each module) is a composite of three images taken through the Pupil Imaging Lens. One is the OSIM's simulated OTE pupil with the 18 primary mirror segments and secondary mirror structure; the other two show the DHS grism apertures for the two DHS sets (differing by 60 degrees in roll) in each module. The images demonstrate that each grism segment samples only two primary mirror segments, for measuring their relative piston. (This is the critical alignment noted in the pupil roll discussion in Section 4.1 above.) Figure courtesy of Douglas Kelly and the NIRCam team.

\subsubsection{NIRSpec}

Finally, we briefly highlight some results of the NIRSpec test campaign, which is reported in more detail in this volume by Birkmann et al. (2016) $)^{4}$, Te Plate et al (2016) ${ }^{11}$, and Giardino et al. (2016) ${ }^{19}$. Trending measurements confirmed good health through the environmental test program for mechanisms (start-up current, position sensors), lamps (flux and stabilization time), and the detectors (confirming the excellent performance of the new SCAs, already tuned to the ASICs at the subsystem level). For the detectors, the superior noise performance (and reduced 1/f striping) of the socalled IRS2 readout mode ${ }^{21}$ showed its value in making weaker features much more visible. Some cross-talk was observed for very bright sources; this was rectified for the "hot op" case $(42.8 \mathrm{~K})$ but requires an adjustment for the "cold op" case (38.5K) during telescope-level testing next year at the Johnson Space Center.

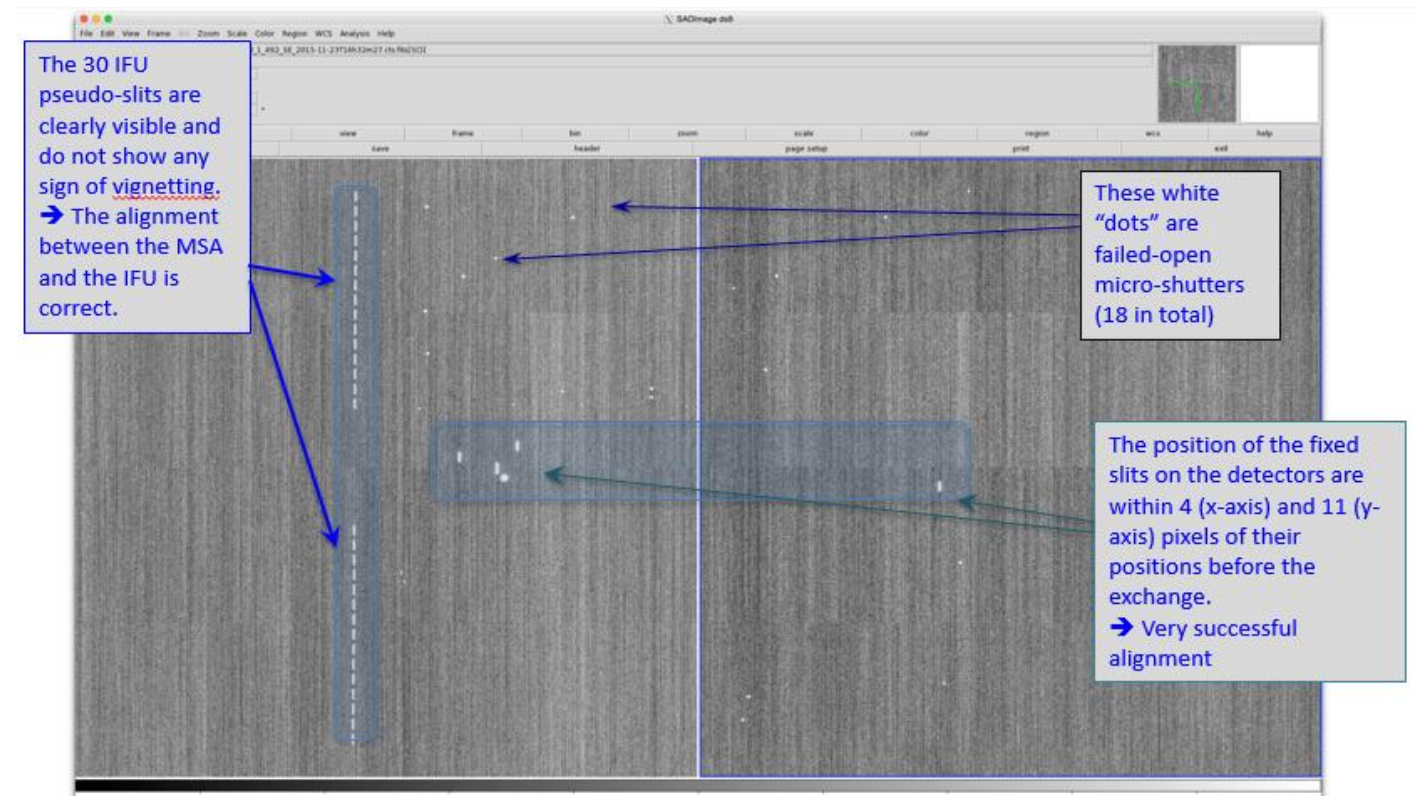

Figure 13. CV3 demonstrated the excellent alignment of the new MSA and detector, with the IFU, fixed slits, and field edges properly registered. Figure courtesy of Stephan Birkmann and the NIRSpec team. 
CV3 data confirmed the accuracy of the installation of the new MSA and focal plane, demonstrating their proper focus and the lack of vignetting for the IFU (Figure 13). The MSA quadrants demonstrated good overall health and performance, though they did show an increase in the number of failed-closed shutters, presumably caused by the ISIMlevel acoustic test program. The MSA still meets requirements, and future acoustic tests are expected to produce less acoustic load on this sensitive item.

A comprehensive program of internal calibration exposures was executed to support the development of a revised optical model, along with acquiring the accompanying spectroscopic flat fields. Data were taken with 36 different MSA configurations and all dispersers, with one long MSA slit per configuration for the gratings, and with two long slits per configuration for the lower-dispersion prism. Dedicated exposures were also taken for the IFU. The result will be a full set of spectroscopic flats for the science data pipeline.

Another interesting observation with NIRSpec was the acquisition of spectra from the external OSIM diffuser. The smooth blackbody nature of these spectra confirmed the absence of significant molecular contamination anywhere in the instrument, a significant result confirming the success of the condensation-avoiding protocols of the ISIM cooldown.

\section{SUMMARY}

The execution of the final ISIM-level cryo-vacuum verification and calibration test, CV3, was a huge milestone in the JWST program. Its successful outcome paved the way for the delivery of the ISIM element, with its powerful suite of scientific instruments, for integration with the rest of the observatory. Integration with the Optical Telescope Element (mounting of the ISIM Prime cold region to the backplane support structure of the telescope) has already occurred (Figure 14).

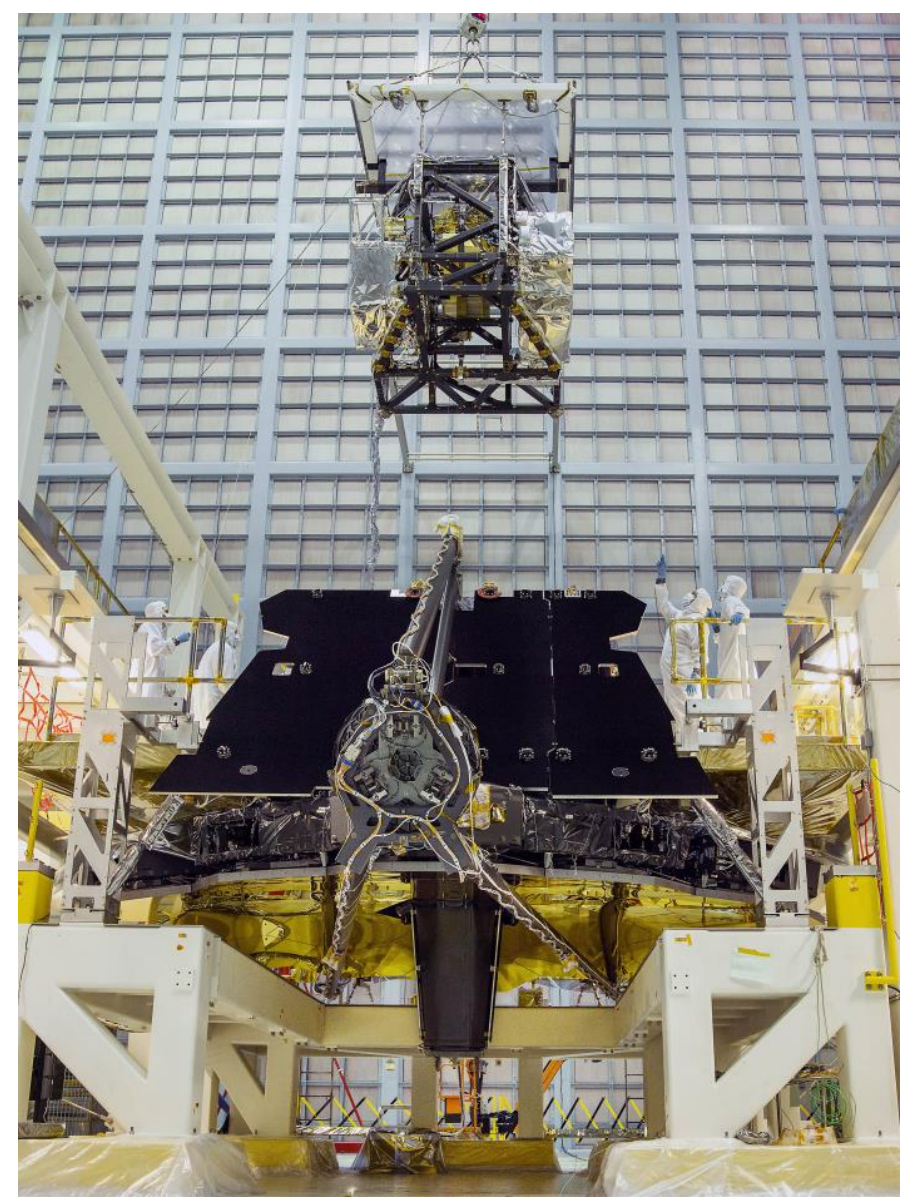

Figure 14. Integration of the ISIM instrument suite into the backplane of the JWST Optical Telescope Element (whose gold-coated Primary Mirror Segment Assemblies are seen facing the floor), after the successful completion of CV3. 


\section{ACKNOWLEDGMENTS}

The JWST program is a collaboration of the National Aeronautics and Space Administration, the European Space Agency, and the Canadian Space Agency. The authors gratefully acknowledge all of the incredibly dedicated members of the CV3 test team and the GSFC facilities team for their expert and unflagging support of the grueling, but highly successful, cryo-vacuum test campaign described above.

\section{REFERENCES}

[1] Rieke, M., Kelly, D. and Horner, S., "Overview of James Webb Space Telescope and NIRCam's Role," Proc. SPIE 5904, 1-8 (2005).

[2] Beichman, C. A., Rieke, M., Eisenstein, D., Greene, T. P., Krist, J., McCarthy, D., Meyer, M., and Stansberry, J., "Science opportunities with the near-IR camera (NIRCam) on the James Webb Space Telescope," Proc. SPIE 8442, $84422 \mathrm{~N}(2012)$.

[3] Bagnasco, G. et al., "Overview of the near-infrared spectrograph (NIRSpec) instrument on-board the James Webb Space Telescope (JWST)," Proc. SPIE 6692, 66920M (2007).

[4] Birkmann, S. M. et al., "The JWST/NIRSpec instrument: update on status and performances," Proc. SPIE 9904, in press (2016).

[5] Rieke, G. H. et al., "The Mid-Infrared Instrument for the James Webb Space Telescope, I: Introduction," PASP Vol. 127, Issue 953, 584-594 (2015).

[6] Wright, G. S. et al., "The Mid-Infrared Instrument for the James Webb Space Telescope, II: Design and Build," PASP Vol. 127, Issue 953, 595-611 (2015).

[7] Doyon, R. et al., "The JWST Fine Guidance Sensor (FGS) and Near-Infrared Imager and Slitless Spectrograph (NIRISS)," Proc. SPIE 8442, 84422R (2012).

[8] Maszkiewicz, M., Saad, K., Rowlands, N., Doyon, R. and Hutchings, J. B., "JWST Fine Guidance Sensor and NearInfrared Imager and Slitless Spectrograph," Optical Payloads for Space Missions (ed S.-E. Qian), ch35, (2015).

[9] Greenhouse, M. A., "Status of the James Webb Space Telescope science instrument payload," Proc. SPIE 9904, in press (2016).

[10] Rauscher, B. J. et al., "Commentary: JWST near-infrared detector degradation - finding the problem, fixing the problem, and moving forward," AIP Advances 2, 021901 (2012).

[11] Te Plate, M. et al., "Getting JWST's NIRSpec back in shape," Proc. SPIE 9904, in press (2016).

[12] Glazer, S., and Comber, B., "James Webb Space Telescope Integrated Science Instrument Module Thermal Vacuum/Thermal Balance Test Campaign at NASA's Goddard Space Flight Center," 46 ${ }^{\text {th }}$ ICES Conference, Paper \#396, in press (2016).

[13] Sullivan, J. et al., "Manufacturing and integration status of the JWST OSIM optical simulator," Proc. SPIE 7731, $77313 \mathrm{~V}(2010)$.

[14] Sullivan, J. et al., "JWST's optical telescope simulator for verification of the Integrated Science Instrument Module," Proc. SPIE 9951, in preparation (2016).

[15] Antonille, S. R. et al., "Optical testing and verification methods for the James Webb Space Telescope Integrated Science Instrument Module,” Proc. SPIE 9951, in preparation (2016).

[16] Rohrbach, S. O., Kubalak, D. A., Gracey, R. S., Sabatke, D. S., Howard, J. M., Telfer, R. C., and Zielinski, T. P., "Critical science instrument alignment of the James Webb Space Telescope (JWST) Integrated Science Instrument Module (ISIM)," Proc. SPIE 9951, in preparation (2016).

[17] Aronstein, D. L., Smith, J. S., Zielinski, T. P., Telfer, R., Tournois, S. C., Moore, D. B., and Fienup, J. R., "Wavefront-error performance characterization for the James Webb Space Telescope (JWST) Integrated Science Instrument Module (ISIM) science instruments," Proc. SPIE 9904, in press (2016).

[18] Rowlands, N., Beaton, A., Chayer, P., Haley, C., Midwinter, C., Volk, K., Warner, G., and Zhou, J., "Updated cryogenic performance test results for the flight model JWT fine guidance sensor," Proc. SPIE 9904, in press (2016).

[19] Giardino, G., "The spectral calibration of JWST/NIRSpec; results from the recent cryo-vacuum campaign (ISIMCV3)," Proc. SPIE 9904, in press (2016).

[20] Bright, S., "MIRI/JWST detector characterization,” Proc. SPIE 9904, in press (2016). 
[21] Rauscher, B. J., Arendt, R. G., Fixsen, D. J., Lander, M., Lindler, D., Loose, M., Moseley, S. H., Wilson, D. V., and Xenophontos, C., "Reducing the read noise of HAWAII-2RG detector systems with improved reference sampling and subtraction (IRS2)," Proc. SPIE 8453, 84531F-84531F-11 (2012). 